\title{
Annette Zgoll \\ Wie der erste Tempel auf die Erde kommt
}

\author{
Der Mythos INNANA BRINGT DAS HIMMELSHAUS FÜR DIE ERDE
}

\author{
Pascal Attinger, dem Grundlagenforscher des Sumerischen ${ }^{1}$ \\ voller Dank für die großzügig geteilten Erkenntnisse, \\ mit den besten Wünschen für die Zukunft ${ }^{2}$
}

\begin{abstract}
It is a great adventure: bringing the first temple out of heaven. The master plan is devised by Innana, and multiple gods carry out the scheme: Innana, her brother Utu and other gods, against the will of the god of heaven, An. This is the outline of the myth named INNANA BRINGS THE HOUSE OF HEAVEN FOR EARTH, told in the epic format of a hymn to Innana. In order to bring the house of heaven for earth, Innana must expose herself to great danger: she must defeat the cosmic Scorpion Gatekeeper and drink up the entire contents of the river that forms the boundary to the underworld.

Innana's actions have fundamental cosmic consequences for gods and humans. The first temple comes into existence: this temple is heaven on earth! In fact, it is the arrival of the temple which creates space (Sumer and the earth) and time (day and night). The message of this myth is especially important from the perspective of the first great city of the ancient world, Uruk.
\end{abstract}

\footnotetext{
Anmerkung: Dieser Beitrag ist im Rahmen der durch das Niedersächsische Ministerium geförderten, deutsch-israelischen Forschungsgruppe TEMEN (= Topography - Mythology - Narration), d. h. in Zusammenarbeit mit Nathan Wasserman und einem Team aus Mitarbeiter/innen der Hebrew University Jerusalem und der Universität Göttingen entstanden. Profitiert hat er auch von der Zusammenarbeit innerhalb der DFG-Forschungsgruppe 2064 „STRATA - Stratifikationsanalysen mythischer Stoffe und Texte in der Antike“. Die Zusammenarbeit in diesen Forschungsverbünden wären nicht denkbar ohne die jahrelange Grundlagenforschung im Göttinger Collegium Mythologicum, wo neue Forschungsansätze langfristig und nachhaltig diskutiert wurden und werden. Den genannten Mitforschenden und finanziellen Förderern bin ich dankbar verbunden. Anja Piller, Jan Steyer und besonders Katharina Ibenthal haben Recherchen zu diesem Beitrag durchgeführt; Klaus Wagensonner hat Photos der Tafel CBS 1531 zur Verfügung gestellt; Frans Wiggermann hat mir erlaubt, seine Umzeichnung eines Rollsiegels wiederzugeben. Ihnen gilt mein herzlicher Dank.
}

1 Vgl. z. B. Attinger 1993, Attinger 2006, Attinger 2019, Attinger 2019a und das monumentale Werk Attinger 2021 mit Dokumenten zur Lesung der Keilschriftzeichen, zur sumerischen Lexik und Grammatik und zu Bearbeitungen sumerischer Texte. 


\section{Uruk als prototypische Stadt}

Es gibt Städte, die mehr sind als Städte. Jerusalem und Rom, Mekka und Bagdad sind solche Städte, hinter denen man mehr als nur eine Ansammlung menschlicher Wohn- und Arbeitsstätten sieht. Für die mesopotamische Antike ist zweifellos Uruk mehr als nur irgendeine Stadt ${ }^{3}$ : Uruk gilt als die Stadt der Innana (A. Zgoll 2012) und ihrer großen Feste (Boehmer 2014), als der Ort, zu dem Innana die göttlichen Machtmittel aus dem Abzu gebracht hat (Farber-Flügge 1973) 4 . Uruk gilt als die Stadt, deren Herrscher Enmerkara, Innanas Auserwählter (Wilcke 2012, 23-25), die Schrift erfunden hat (Mittermayer 2009). Uruk ist die Metropole, in der Gilgameš geherrscht und für die er die unvergängliche Stadtmauer ${ }^{5}$ erbaut hat (A. Zgoll 2010).

Dass Uruk als herausragende Stadt angesehen wurde, hängt, wie sich in diesem Beitrag zeigen wird, insbesondere mit einer Institution und ihrer mythischen Geschichte zusammen: mit dem Tempel E-ana. Wie sich zeigen wird, ist nämlich dieser Tempel mehr als irgendein Tempel. Vielmehr handelt es sich der mythischen Überlieferung zufolge um einen Tempel, der vom Himmel auf die Erde gekommen ist ...

2 Der Beitrag ist ein Addendum zur Festschrift für Pascal Attinger (Mittermayer/Ecklin 2012).

3 Vgl. Crüsemann/van Ess/Hilgert/Salje 2013.

4 Eine andere Version dieses Mythos wird im Text Enmerkara und der Herr von Arata (Mittermayer 2009) erzählt, wo Innana den Enmerkara schickt, um diese numinosen Machtmittel vom Gott Enki „abzuholen“; offenbar erhält er sie dort als Geschenk. - Die Variante des Mythos INNANA BRINGT DIE NUMINOSEN MACHTMITTEL VON ERIDU NACH URUK, die in der textlichen Konkretion des Preisliedes Innana und Enki erzählt wird, dürfte für Aufsehen gesorgt haben, da in anderen Mythen gerade Enki derjenige ist, der durch seinen Einfallsreichtum die Dinge für die Menschen zum Guten bewegt. Hier im Mythos INNANA BRINGT DIE NUMINOSEN MACHTMITTEL VON ERIDU NACH URUK zeigt sich die Stadtgöttin von Uruk in genau dieser Rolle: Trickreich kann sie für ihre Stadt das Notwendige gewinnen. - Wenn in verschiedenen Mythen(versionen) verschiedene Protagonisten als verantwortlich gezeigt werden, dass sie wesentliche Güter für die Erde und die Menschheit oder für eine spezifische Stadt oder Gruppe erschaffen oder zugänglich machen, dann lässt dies auf verschiedene Gruppierungen schließen, für welche die jeweilige Mythenversion wichtig ist. So zeigt sich im Mythos INNANA BRINGT DIE NUMINOSEN MACHTMITTEL VON ERIDU NACH URUK eine Überlieferung, die aus Uruk stammt; in Eridu hätte man diesen Mythos zu bestimmten Zeiten gar nicht oder in ganz anderer Akzentuierung, also in einer anderen Version erzählt.

5 Zugleich wird Uruk mit seiner Stadtmauer zur Metapher für das Gilgameš-Epos selbst, das damit ebenfalls Unsterblichkeit für sich beansprucht (vgl. A. Zgoll 2010). 


\section{INNANA BRINGT DAS HIMMELSHAUS FÜR DIE ERDE: Textgrundlage}

Wichtigster Kronzeuge zu diesem Thema ist ein Preislied auf die Göttin Innana, das inhaltlich betrachtet einen mythischen Erzählstoff bearbeitet, formal betrachtet mit einem umfänglichen episch-erzählenden Hauptteil und einem hymnisch-preisenden Abschluss gestaltet ist ${ }^{6}$. Dieses Preislied ist erst seit gut 20 Jahren in einer ersten Edition bekannt geworden. Es wird als Innana raubt den großen Himmel bzw. Innana und An bezeichnet. Im Folgenden werde ich zwischen diesem Text und dem in ihm verarbeiteten Mythos, d. h. dem mythischen Erzählstoff unterscheiden. Als Bezeichnung für den Text würde man idealiter wie in der Antike - die Anfangswörter (plus modernen Titel) wählen. Da der Textbeginn bislang unbekannt ist, wähle ich eine zentrale Phrase des Textes, nämlich an gal karede, was bedeutet ,um den großen Himmel wegzunehmen“. Als Textbezeichnung dient mithin an gal karede / Innana und An ${ }^{7}$. In die Bezeichnung des Mythos, der in diesem Text hauptsächlich verarbeitet wird ${ }^{8}$, fließen die Ergebnisse der vorliegenden Studie ein; sein Titel lautet: InNANA BRINGT DAS HIMMELSHAUS FÜR DIE ERDE ${ }^{9}$.

Überliefert ist das Epos auf drei teilweise erhaltenen Tontafeln und einem kleinen Fragment einer Tontafel ${ }^{10}$. Die Tafeln datieren mit van Dijk (1998) in die

6 Die Definition von Mythen folgt C. Zgoll, „Grundlagen einer stoffanalytisch-vergleichenden Mythosforschung“ im vorliegenden Band, Abschnitt 1.3; ausführlicher C. Zgoll 2019, 557-563. Im vorliegenden Text an gal karede / Innana und An liegt dem zufolge nicht „ein Mythos“, sondern eine in einer ganz bestimmten textlichen Gestalt konkretisierte Version eines Mythos, d. h. eines mythischen Stoffes vor.

7 Analog etwa zu an galta / Innanas Gang (zur Unterwelt) oder inūma ilū awìlum / Atram-ḩasīs.

8 Mythische Texte aus dem antiken Mesopotamien thematisieren in der Regel nicht nur einen mythischen Erzählstoff, sondern mehrere. Vgl. z. B. A. Zgoll $2020 \mathrm{zu}$ den im epischen Preislied an galta / Innanas Gang verarbeiteten Mythen. Wilcke (1999, 70) bezeichnet das Phänomen als „Mythenkoppelung“ (mit Verweis auf Kirk 1970); um die damit verbundene Assoziation einer einfachen Hintereinanderschaltung zu vermeiden (meist sind die Verquickungen komplizierter), kann man in diesem Zusammenhang auch von „Stoffkonglomeraten“ oder „Stoffkombinationen" sprechen (vgl. C. Zgoll 2019, 160-163).

9 Bezeichnungen von Texten (Texttitel) sind immer durch Kursivierung markiert (auch bei sumerischem Texten), Bezeichnungen von Mythen(versionen) (Mythentitel) sind immer durch Kapitälchen markiert.

10 Auf dieser Basis ist etwa die Hälfte des Textes, der ursprünglich 165 Zeilen umfasste, zu rekonstruieren; 97 Zeilen sind erhalten, aber nicht immer ganz, 68 Zeilen fehlen vollständig. 
erste und zweite Hälfte des 2. Jt. v. $\mathrm{Chr}^{11}$. Sie stammen aus verschiedenen mesopotamischen Städten, Uruk (UkD), Nippur (NiC), vielleicht Sippar (Si $\left.{ }^{?} \mathrm{~A}\right)$, eine ist von unbekannter Herkunft (UnB).

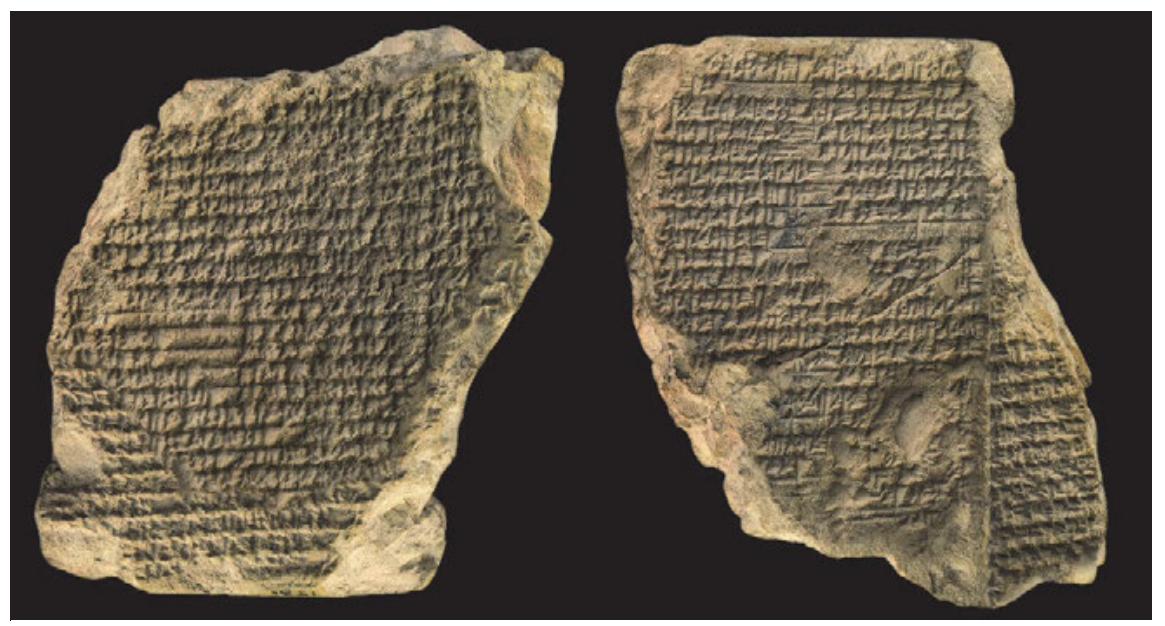

Abb. 1: Tafel Si'A, CBS 1531, heute Philadelphia. Photo Klaus Wagensonner

Pionierarbeit und Grundlage aller weiteren Studien ist die Edition von van Dijk unter der Mitarbeit von Cavigneaux (1998). Cavigneaux hat die Tafeln UnB und $\mathrm{NiC}$ identifiziert und für UnB auch die Umzeichnung angefertigt. Die Lesung der Philadelphia-Tafel Si?A wird jedem, der diese schon einmal in Händen gehalten hat, höchste Bewunderung abverlangen: diese an der Oberfläche durch Abrieb verstümmelte Tafel zu lesen ist eine Entzifferungsleistung ersten Ranges. Auf dieser Studie von van Dijk mit Cavigneaux (1998) basiert eine italienische Übersetzung durch Pettinato (2001) und eine englische Übersetzung des Electronic Text Corpus of Sumerian Literature der Universität Oxford (Black et al. 2005). Zólyomi (2000) konnte die Platzierung des Uruk-Fragments (UkD) klären. 2015 ist eine Übersetzung des Epos durch A. Zgoll erschienen, die auf einer neuen Edition des Textes basiert und den vorliegenden Beitrag ergänzt ${ }^{12}$.

11 Alster (2004, $25 \mathrm{f} \mathrm{Anm.} \mathrm{21)} \mathrm{verweist} \mathrm{darauf,} \mathrm{dass} \mathrm{Texte} \mathrm{mit} \mathrm{zwei} \mathrm{bilingual} \mathrm{Sumerisch-}$ Akkadisch beschrifteten Kolumnen nicht immer kassitisch sind, sondern auch in Texten aus Emar und Ugarit vorkommen.

12 Vgl. A. Zgoll 2015. Die Publikation der neuen Edition ist in Vorbereitung. Sie wird in der Reihe Mythological Studies erscheinen. 
Daneben gibt es Beiträge zu Detailfragen. $\mathrm{Zu}$ einer Passage mit astronomischem Bezug (Z.146 f) haben Brown und Zólyomi (2001) gearbeitet, was aufgegriffen und weitergeführt wurde durch Cancik-Kirschbaum (2005) und Cooley (2013). Richter (2003) stellt in seiner Rezension von van Dijk heraus, dass Innanas Tat nicht auf den Himmel als solchen zielt, sondern auf das Himmelshaus. Alster (2004) hat die Gestalt der Fischergötter näher beleuchtet. Unklar bleibt mir die philologische Grundlage des Textverständnisses bei Annus (2016) ${ }^{13}$.

Im Folgenden wird es darum gehen, die Version des im Text verarbeiteten Mythos zu rekonstruieren und den grundlegenden Anliegen dieser Mythenversion auf die Spur zu kommen.

\subsection{Protagonisten}

Protagonisten im episch überlieferten Mythos sind drei große himmlische Gottheiten. Am wichtigsten ist die Göttin Innana, deren Name, abgeleitet aus * n in 'a n-ak, „Herrin des Himmels“ bedeutet ${ }^{14}$. Sie ist Stadtgöttin von Uruk. Eine ihrer bedeutsamsten Manifestationen ist das Venusgestirn. Ihr Gegenspieler ist der Himmelsgott An, dessen Name seinen Bedeutungsbereich, den Himmel, bezeichnet. Er gilt in diesem Epos als Gemahl der Innana. Als Helfer der Innana wird der Sonnengott, ihr Bruder, eingeführt. Sein Name Utu wird mit dem Zeichen UD geschrieben, das in der Lesung / $\mathrm{u} /{ }^{15}$ auch die Wörter „Sonne“, „Licht“ und „Tag“ bezeichnet. Daneben treten unbekanntere, kleinere Gottheiten als Helfer auf, v. a. ein Gott namens Adag(ki)bir, dessen Aufgabenbereich der himmlische Ozean ist und der auch als Fischer bezeichnet wird; wichtiges Instrument dieses Adag(ki)bir ist sein riesiges Fischernetz. Weitere numinose Helfer und dämonische Gegenspieler werden erwähnt (die Götter HुI.EN.HुI.ŠA, Z. 81, und Sulazida, Z. 129, u du g-Schutzgeister, Z. 130, ein kosmischer Wächter in Gestalt eines Skorpions, Z. 133).

13 Nach Annus (2016) geht es im Text um das Herabkommen der Göttin Innana aus dem Himmel bzw. aus Aratta nach Uruk (a. 0. 31-33 und passim, ohne Diskussion philologischer Grundlagen).

14 Die lautliche Entwicklung von * $n$ in -' a n-a k führt über eine Assimilation des Alephlautes an /n/ zur Lautung /nn/ am Anfang des Namens, d.h. zur Schreibung Innana (beim Genitiv „des Himmels“ (an-ak) liegt hingegen keine Doppelung vor); insofern ist mit Marchesi 2006, 211; Attinger 2007 „Innana“ zu umschreiben.

15 In Umschriften wird dieses Zeichen UD, wenn es die Lesung / $\mathrm{u}$ / hat, zur eindeutigen Identifizierung als $\mathrm{u}_{4}$. $\mathrm{d}$ wiedergegeben. 


\subsection{Skizze des Handlungsverlaufs}

Der Text zeichnet einen klar nachvollziehbaren Bogen: Innana möchte das Eana vom Himmelsgott An bekommen; der aber möchte es ihr nicht geben. Und nun schmiedet sie weitreichende Pläne ${ }^{16}$ mit dem Sonnengott Utu, der schwört, ihr zu helfen, und mit einem Fischerei-Gott, der schrecklichen Stürmen trotzt, damit das E-ana auf die Erde kommen kann. Und es gelingt tatsächlich: Innana „raubt“ das „Himmelshaus“!

Wie dieser Coup im Einzelnen vor sich geht, ist durch den lückenhaften Erhaltungszustand der Tafeln schwer zu sagen. Einer neuen philologischen und mythologischen Analyse vorgreifend lässt sich hier schon festhalten:

Zunächst fährt das E-ana auf dem Himmels-Ozean auf einem riesigen Schiff ${ }^{17}$, wo es von schrecklichen Winden, v. a. den Südstürmen bedroht wird. Dort, in einem Sumpfgebiet, d. h. verborgen vor etwaigen Verfolgern, geht das E-ana in den Fluten unter. Mit einem riesigen Fischernetz gelingt dem Fischergott dann gemäß der Anweisung Innanas die Rettung des Tempels vor dem Untergang im kosmischen Himmelsozean ${ }^{18}$.

16 Anders Alster (2004, 36): "when Inanna wanted to take possession of Eanna, [...] she was prevented from doing so by An, who sent the South wind in order to overthrow her boat. The fisherman Adagbir then found a way to help her from drowning." Diese Interpretation basiert auf der Übersetzung der Zeilen $153 \mathrm{f}$ durch van Dijk (1998), der davon ausgeht, An habe Innana vor dem Ertrinken bewahrt; nach der diesem Beitrag zugrundeliegenden neuen Edition ist hier vielmehr vom Versinken des E-ana die Rede. Auch inhaltliche Gründe sprechen gegen die Deutung Alsters: die ausführlichen Schilderungen von Innanas Plänen zeigen, dass Innana schon im Voraus erwägt, wie alles gelingen kann. Eine solche genaue Planung, die zum Erfolg führt, ist typisch für das, was man sich von der Göttin Innana erzählt; Entsprechendes findet sich im Mythos INNANA BRINGT DIE UNTERWELTSMACHTMITTEL AUF DIE ERDE, der inkorporiert ist in den Mythos INNANA WIRD HERRSCHERIN ÜBER TOD UND LEBEN, was textlich erhalten ist im epischen Preislied angalta / Innanas Gang zur Unterwelt (vgl. A. Zgoll 2020 und A. Zgoll/C. Zgoll 2020). Innana schlittert diesen Mythen zufolge gerade nicht in eine unvorhersehbare Katastrophe, sondern hat die Widrigkeiten im Voraus schon einberechnet und dagegen vorgesorgt.

17 Diese Vorgänge spielen sich im Himmel ab, was sich aus dem Ablauf der Geschehnisse ergibt: Damit Utu das E-ana unter den Horizont bringen kann (Z. 131), muss Innana den Skorpion-Wächter kurzzeitig unschädlich machen, indem sie ihm den Schwanz abschlägt (Z.133) und dann den Signal-Schrei für Utu gibt (Z. 136), dass der Weg frei ist. Utu und Innana sorgen also dafür, dass das E-ana von den Himmelsregionen auf die Erde kommen kann. Da Handlungen des Fischergottes Adagbir (Z.126-128), die zuvor ausführlich geplant wurden (Z. 56-125), den Taten Innanas und Utus vorausgehen (Z.129-138), müssen sie innerhalb des Himmels lokalisiert werden.

18 Anders Alster 2004, 36: Mit einem Netz zähme der Fischergott die Winde und veranlasse, dass Innana sich im Schilfrohr verstecken könne. 
Nachdem das E-ana auf diese Weise vom „Himmelsfundament“ (sumerisch

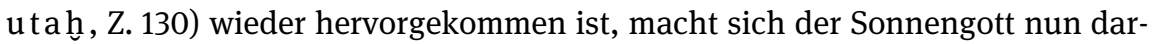
an, das E-ana unter den Horizont zu bringen ${ }^{19}$. Damit dieser kosmische Transfer gelingen kann, muss aber erst einmal einer der streng bewachten Wege frei werden. Die Götter wählen eine schmale Passage (Z.122) am Horizont, die vom astralen Skorpion bewacht wird ${ }^{20}$.

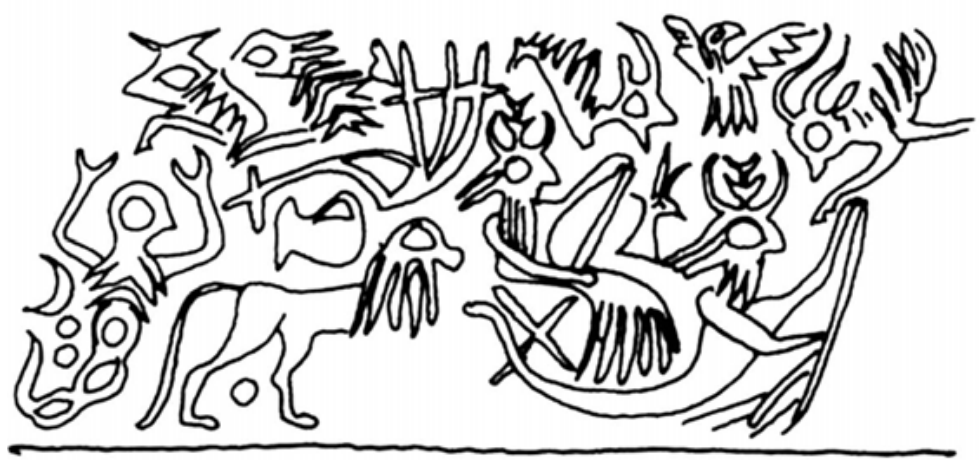

Abb. 2: Rollsiegel vom Anfang 3. Jt. mit Utu und Bootsgott (von der Osten 1934, Nr. 47), Umzeichnung Wiggermann 2013, 121; ebd. 127 Hinweise zur Interpretation des Rollsiegels und der Vermerk, dass die Bedeutung des Bildes bislang nicht eindeutig ist. Der aus an gal karede / Innana und An rekonstruierbare Mythos INNANA BRINGT DAS HIMMELSHAUS FÜR DIE ERDE und die Abbildung auf diesem Rollsiegel können sich hier gegenseitig erhellen: Der Sonnengott fährt auf einem Boot(sgott) über den Himmelsozean. Den Übergang in andere kosmische Bereiche bewacht der Skorpionwächter mit erhobenen Armen.

Dieser Skorpion wird von Innana attackiert: Innana hackt dem Wächter der Himmelstore dabei den Schwanz ab. Damit wird - so meine Interpretation kurzfristig der Weg von den himmlischen Regionen zur Erde frei. Innana brüllt

19 Vgl. Wiggermann 2013, 127.

20 Vgl. van Dijk 1998, 27 ad Z. 133 zum Skorpion; vgl. auch Wiggermann (2013, 126 f): „Der Skorpionmensch, der im Gilgameš-Epos das Zwillingsgebirge am Horizont bewacht, hat einen Vorläufer im Skorpionmenschen am Eingang der Totenwelt und in einem noch etwas älteren Skorpion mit astraler Funktion. Der Letztere tritt in einer im 3. Jt. sehr beliebten Szene auf, für die es bislang keine allgemein akzeptierte Deutung gibt. [...] Der astrale Skorpion weist den Weg zu einer Deutung der Szene als Reise der Sonne über einen kosmischen Ozean, unterirdisch oder oberirdisch“. Zum astralen Skorpion vgl. auch Zernecke 2008, 107-127. 
löwengleich (Z. 134) $)^{21}$, was dem Sonnengott das Signal gibt, um in diesem Moment das E-ana „fallen zu lassen“, damit es auf die Erde gelangen kann (Z. $135 \mathrm{f})$.

Wie auch immer das Abenteuer noch zu präzisieren sein mag - am Ende jedenfalls ist der Plan Innanas aufgegangen, das E-ana ist tatsächlich auf Erden. Und der Himmelsgott entscheidet sogar dem Tempel sowie der Göttin eine gute Zukunft ${ }^{22}$. Das Textende offenbart den Text dann als Preislied auf Innana und ihre Tat:

\begin{tabular}{|c|c|}
\hline${ }^{163} E_{2}-a n-n a \cdot k$ an-da im-da- & ${ }^{163}$ Weil du (Innana) dabei bist, das E-ana \\
\hline 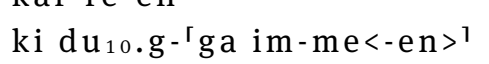 & 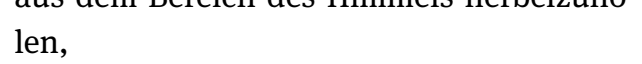 \\
\hline & $\begin{array}{l}\text { weil du dabei bist } \mathrm{zu} \text { sagen "Es <ist> das } \\
\text { (Haus), das die Erde gut gemacht hat!", }\end{array}$ \\
\hline $\begin{array}{l}{ }_{b a^{23}}^{164} \text { nun-gal-e-ne diri.g-ga- } \\
\text { ba }^{23}\end{array}$ & $\begin{array}{l}{ }^{164} \text { nachdem du die großen Fürsten(götter) } \\
\text { (= Anuna) überragt hast, }\end{array}$ \\
\hline $\begin{array}{l}{ }^{165} \mathrm{ki}-\mathrm{sikil}{ }^{\mathrm{d}} \text { Innana.k } \\
\mathrm{za}_{3}-\mathrm{mim}-\mathrm{zu} \mathrm{mah}_{-\mathrm{m}_{3}}\end{array}$ & $\begin{array}{l}165 \text { (o) junge Frau Innana: (Dieses) Preislied } \\
\text { auf dich ist machtvoll! }\end{array}$ \\
\hline
\end{tabular}

Das Problem, das sich hieraus ergibt, lässt sich einfach auf den Punkt bringen: Wieso wird Innana, wenn sie ein so gemeiner Räuber ist und dem Himmelsgott sein Himmelshaus raubt, am Ende desselben Textes von eben diesem Himmelsgott erhöht und bestätigt und wieso endet der Text mit einem überschwänglichen ${ }^{24}$ Lobpreis? Die folgenden Kapitel werden sich diesen Problemen des mythischen Epos aus verschiedenen Perspektiven annähern.

21 Van Dijk (1998, 22) versteht dieses Brüllen als Schmerzensschrei des getroffenen Skorpions. Die Schreibung der Verbalform b a - ni - in - r a mit Ergativ der Personenklasse macht allerdings deutlich, dass es Innana ist, die den Schrei ausstößt. Dieser hat die Funktion eines Signals für Utu, wie es in der Planung schon abgesprochen worden war. Dass dieser Schrei wie der eines Löwen ist, passt genau zu Innana, deren Tier der Löwe ist. Ihr Gebrüll wird auch in anderen Texten im Kontext des Niederschlagens von Feinden beschrieben, vgl. Fink 2017.

22 Die Festlegung des An beinhaltet, dass der Tempel fest wie der Himmel sein wird und dass die Menschheit der Innana unterworfen sein wird, zwei bemerkenswerte und weitreichende Aussagen.

23 Komposittext aus dem Textzeugen NiA und UnB.

24 Der Lobpreis am Ende des Textes ist in vielfältiger Hinsicht herausragend: (1) Ein SchlussPreis ( $\left.\mathrm{za}_{3}-\mathrm{mim}\right)$ wird oft als „gut“ beschrieben - das hier gewählte „großartig, erhaben, machtvoll“ (mah) ist mehr. (2) Ein Schluss-Preis ist üblicherweise in der 2. Person gehalten; hier hingegen findet sich zunächst ein Preis in der 3. Person (Z.159-162), der dann in einen Preis in der 2. Person mündet (Z. 163-165). (3) Ein Schluss-Preisvermerk umfasst in anderen 


\section{Häuser im Himmel}

\subsection{Das E-ana als „Himmelshaus“}

Der sumerische Name E-ana bedeutet „Haus des Himmels(gottes An)“ und zugleich, mit Genitivus loci, „Haus im Himmel“25. Dass es im Himmel Wohnstätten der himmlischen Götter, „Himmelshäuser“, gibt, ist gut bekannt ${ }^{26}$; besonders deutlich wird es im akkadischen Enūma elîš / Weltschöpfungsepos von Marduks Erhöhung, wo der Himmel als Ašrata bezeichnet wird, was mit Gabriel ${ }^{27}$ als sumerisiertes ašrāti, ein akkadisches Wort für „Heiligtümer“ zu verstehen ist. Der Himmel besteht aus Heiligtümern, nämlich den „Himmelshäusern“, wo die himmlischen Gottheiten wohnen.

\subsection{Ein Tempel muss aus einem numinosen Raum auf die Erde kommen}

All diese Götterwohnungen im Himmel haben Teil an der Substanz des Himmels, d. h. zugleich des Himmelsgottes An. So jedenfalls kann man ein mythisches Hylem ${ }^{28}$ verstehen, das sich im Kontext religiös-hymnischer Texte über

mythischen Epen oft eine Zeile oder zwei Zeilen, hier hingegen finden sich sieben Zeilen! Insgesamt lässt sich festhalten, dass ein solches Preislied machtvoll ist, insofern es die gepriesenen Eigenschaften auf den Gepriesenen überträgt bzw. sie verstärkt, vgl. A. Zgoll 2012a, $27 \mathrm{f}$ am Beispiel der Keš-Hymne und vgl. auch Gerstenberger 2018; ausführlich wird dies in dem in Vorbereitung befindlichen Buch über Religion in Mesopotamien dargestellt werden.

25 Zum Genitivus loci vgl. Jagersma 2010, 148, Bsp. 50: $\mathrm{e}_{2}$-gal urub ${ }^{\mathrm{ki}}$-ka-ni „sein Palast in Urub“.

26 Vgl. Heimpel 1986, 130-132; vgl. z. B. auch die Schilderung, wie Etana in ein Gotteshaus im Himmel gelangt (vgl. Haul 2000) oder den Einzug der Göttin Innana, vorbei an den Torhütergottheiten, in die himmlischen Wohnungen im sumerischen Lied nin-me-šara / Innana B, vgl. A. Zgoll 2015a (mit weiterer Literatur) und 2021.

27 Gabriel 2014, 150.

28 Ein Hylem ist die kleinste handlungstragende Einheit eines Erzählstoffes. Solche Hyleme sind wichtig für die Rekonstruktion von Mythen: Mythen und andere Erzählstoffe lassen sich als Sequenzen solcher Hyleme rekonstruieren. Vgl. dazu C. Zgoll, „Grundlagen der hylistischen Mythosforschung“ im vorliegenden Band, Kapitel 2 und ausführlich C. Zgoll 2019. Exemplarische Anwendungen der Methodik aus verschiedenen Disziplinen finden sich in den Beiträgen dieses und des von A. Zgoll/C. Zgoll 2020a herausgegebenen Bandes. Für englische Darstellungen dieser Rahmentheorie und Methodik der Mythosforschung vgl. C. Zgoll 2020 und A. Zgoll 2020a. 
Tempelbauten immer wieder findet: Dass nämlich der Himmelsgott die Tempel gegründet hat ${ }^{29}$. Die Aussage besagt nicht weniger, als dass ein solcher Tempel uranfänglich im Himmel existiert. Nehmen wir als Beispiel das Tempelbauprojekt eines berühmten Herrschers des 21. Jh. v. Chr. namens Gudea („Berufener“). Bevor er einen Handgriff zum Bau auf Erden tun kann, existiert dieser Tempel nach mesopotamischer Vorstellung längst im Himmel: Sein Stadtgott, der ihn zum Bau auf Erden beauftragt, bezeichnet den Tempel als

$\mathrm{e}_{2}-\hat{\mathrm{g}} \mathrm{u}_{10} \mathrm{E}_{2}-\mathrm{ninnu}$ An-ne

ki ĝar-ra
„Mein Haus E-ninnu, das (der Himmelsgott) An gegründet hat“

Gudea Zylinder A 9:1130

Mit einer derartigen Formulierung identifiziert man den auf Erden gebauten Tempel mit dem von An im Himmel gegründeten Gotteshaus. Anders ausgedrückt, die Bezeichnung des Tempels als „Haus, das An gegründet hat“ trägt die Botschaft, dass der Tempel ein Himmelshaus ist. Solche von An gegründeten oder anders ausgedrückt, im Himmel begründeten Tempel müssen auf die Erde kommen: Innanas Tat zeigt eine prototypische Möglichkeit, wie man ein solches Geschehen beschreiben konnte. Ein ähnliches Konzept findet sich z.B. beim Tempel in Eridu, dessen Gott Enki ihn im Süßwasserozean Abzu erschafft und von dort auf die Erde bringt. So heißt es in u re'a / Enkis Fahrt nach Nippur 9 preisend von Enki, dass er derjenige ist, der den strahlenden Bezirk, also den Tempel, kunstvoll geschaffen und aus dem Abzu hervorgebracht hat (mušs $\mathrm{ku}_{3}$ galam du 11- $\left._{\text {ga abzu-ta }} \mathrm{e}_{3}-\mathrm{a}\right)$.

29 Gemeint ist damit nicht, dass er einen Tempel auf Erden gegründet hat. Auch wenn die idiomatische Wendung „gründen“ ki - - ĝ ar mit den Bestandteilen ki „Erde“ und ĝ ar „setzen“ das zunächst zu implizieren scheint; doch ki - - ga a ist im Sumerischen idiomatisch, d. h. die Summe der Teile ki und $\hat{g}$ ar bedeutet insgesamt „gründen“. Für eine alternative Interpretation vgl. den Beitrag von Kärger, Götter, Tempel, numinose Machtmittel ..., Abschnitt 4.2.2 im vorliegenden Band.

30 Die Aussage wird in Gudea Zylinder A 27:8 und B 20:20 variierend wiederaufgenommen. 


\section{Vom Himmel auf die Erde: Der Transfer}

\subsection{Wie kommt ein Himmelshaus auf die Erde? Aktivierung, Identifizierung und Transfer}

Welche Modelle der Vermittlung zwischen Himmel und Erde sind denkbar? Drei mögliche Richtungen lassen sich mit den Schlagwörtern Aktivierung, Identifizierung und Transfer angeben. Zur Aktivierung von Tempeln forscht eine Göttinger Projektgruppe $^{31}$. Knapp auf den Punkt gebracht bezeichnet Aktivierung von Tempeln das Phänomen, dass die Erschaffung eines Tempels in mesopotamischen Quellen nach Abschluss der Bautätigkeiten nicht abgeschlossen ist. Vielmehr - so die Ergebnisse unserer Forschungen - ist es entscheidend, dass der Tempel nach seiner materiellen Fertigstellung für seine Aufgaben mit besonderen Fähigkeiten ausgestattet wird. Diese Befähigung des Tempels vollzieht sich nach verschiedenen sumerischen Quellen $-\mathrm{u}$. a. dem Preislied auf Nintu für das Heiligtum von Keš (nam nune / Keš-Hymne) ${ }^{32}$ oder dem Preislied auf Enki für das Heiligtum von Eridu (u re'a / Enkis Fahrt nach Nippur) ${ }^{33}$ - durch den Lobpreis des Tempels durch eine oder mehrere Gottheiten. Durch die machtvollbeschwörenden Worte eines solches Preises wird dem Tempel durch die Götter seine besondere Wirkmacht „eingesprochen“, so dass der Tempel diese Macht dann besitzt ${ }^{34}$.

Die Identifizierung von Tempeln ist aufs engste mit der Aktivierung verzahnt; um diese Identifizierung wird es in Abschnitt 7.1 und 7.2 gehen. Zunächst also zum Transfer zwischen Himmel und Erde: Wenn Tempel wirklich Himmelshäuser sind, dann sollte sich irgendwo etwas über diesen Transfer finden lassen.

31 Konzepte zur Aktivierung von Tempeln und anderen Entitäten (wie Gottheiten oder die für das gesamte Universum wichtige kosmische Hacke) im antiken Mesopotamien untersucht die Projektgruppe von A. Zgoll, B. Kärger und A. Merk, vgl. http://www.uni-goettingen.de/de/41 0999.html. Ein Verweis auf die Macht von Preisliedern findet sich in A. Zgoll 2012a, 27 f; in eine ähnliche Richtung geht auch Gerstenberger 2018. Eine ausführliche Darstellung wird A. Zgoll in der Monographie zu Religion in Mesopotamien geben (in Vorbereitung).

32 Eine Edition durch Brit Kärger, Göttingen, ist in Vorbereitung.

33 Passender ist für diesen Text der Titel Enkis Tempelstiftung (oder ähnlich). Die neueste Bearbeitung ist Ceccarelli 2012. Eine Edition durch Anja Merk, Göttingen, ist in Vorbereitung.

34 Als Pendant zu diesem „Ein-Sprechen“ durch Preislieder fungiert in Ritualtexten auch sumerisch šid/akkadisch manû, was nicht einfach „rezitieren“, sondern vielmehr „etwas in etwas ein-sprechen“, „,be-sprechen“ bedeutet, vgl. A. Zgoll 2006, 386-391. 
Ein sumerisches Epos über den frühen König Gilgameš löst das Problem des Transfers vom Himmel auf die Erde in folgender Weise ${ }^{35}$ :

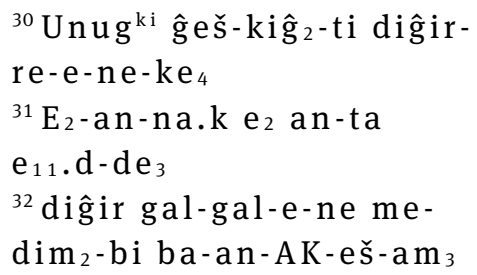

32 Es ist so, dass die großen Götter ${ }^{30}$ für Uruk, die Werkstätte der Götter, 31 für das E-ana, das Haus, das aus dem Himmel heruntergebracht wird, ${ }^{32}$ dessen Glieder (oder: Schiffsplanken ${ }^{36}$ ) gemacht haben.

Gilgameš und Akka Z. 30-32 37

Könnte das bedeuten, dass die großen Götter das Himmelshaus E-ana mit Gliedmaßen ausgestattet haben, die ihm eine Bewegung vom Himmel auf die Erde erlauben? Oder ist das E-ana hier vielmehr in Gestalt eines Schiffes vorgestellt?

Was an dieser Stelle unproblematisch klingt, erhält in einem mythischen Epos vom König Etana ein anderes Gewicht. Dort heißt es am Anfang, dass die Heiligtümer noch nicht erbaut und die Tore (des Himmels) verriegelt sind ${ }^{38}$. Hier ist es demnach (noch) nicht möglich, die Himmelshäuser auf die Erde zu holen (oder selbst in den Himmel zu gelangen, was Etana später gelingen wird). Ein intensives Studium des Epos an gal karede / Innana und An (vgl. Abschnitt 5) und die Rekonstruktion des darin verarbeiteten Mythos (vgl. Abschnitt 6) führt $\mathrm{zu}$ der Entdeckung, dass dieser mythische Text vom Prototyp eines solchen Transfers berichtet.

35 In Gilgameš und Akka wird Uruk als ki-tuš mah An-ne $\mathrm{e}_{2}$ ĝar-ra-ni, ,sein (= Ans) erhabener Wohnort, den An gegründet hat“ (Z. 109) bezeichnet, wobei ĝ a r abgekürzt für ki - $\hat{\mathrm{g}}$ a r steht; alternativ, fast synonym, kann man auch übersetzen „den An aufgestellt hat“.

36 Den Hinweis auf diese andere mögliche Bedeutung von me-dim $\mathrm{m}_{2}$ und Belege dazu verdanke ich Ingo Schrakamp. Als „(Schiffs-)Planken“ o. ä. wird me- d i m 2 sonst für gewöhnlich mit dem Determinativ für Gegenstände aus Holz ( $\hat{\mathrm{g}}$ e š) versehen. Zu m e-d i m 2 vgl. Selz 1998, 261 Anm. 34 „Seitenplanken“. Eine Diskussion der Stelle mit Forschungsliteratur und leicht abweichender Übersetzung findet sich in Anhang 9 des Beitrages von Kärger, Götter, Tempel, numinose Machtmittel ... in diesem Band.

37 Vgl. Katz 1993, Wilcke 1998, George 1999, Neumann 2013, Waetzold 2015.

38 Etana-Epos, altbabylonische Version 1:9 f lā banû ištīniš parakkū / sebetta bābū uddulū elu dapnim - „(Noch) nicht erbaut waren sämtlich die Heiligtümer. / Siebenfach waren die Tore (gemeint: des Himmels) gegen den Mächtigen verriegelt.“ Vgl. Haul 2000, 106 f mit 122 f. Mit Wilcke 1977, 157 verstehe ich sebetta adverbiell und gehe anstelle eines „rare and archaic grammatical feature“ (Lambert 1980) davon aus, dass die Wortstellung in einem derart poetisch gestalteten Text freier gehandhabt wird als in Alltagstexten. - Für eine jüngere Version der Passage vgl. Kinnier Wilson 2007, 13-15. 


\subsection{Vom Himmel auf die Erde: Der Transfer als „Raub“}

So viel in an gal karede / Innana und An auch noch fehlen oder unklar sein mag, eines ist ganz klar: Es ist Innana, die hier das Himmelshaus E-ana auf die Erde holt. Und dieses Geschehen wird als „Raub“ bezeichnet. Das Verbum kar „wegnehmen, rauben“ erscheint neunmal im erhaltenen Text, ist also ein Kernbegriff. So spricht z. B. Innana davon, das E-ana zu „rauben“, als sie versucht, ihren Bruder, den Sonnengott Utu, als Helfer für ihre Pläne zu gewinnen. Ihm sagt Innana:

[an] me-en-de - na ki meen-de $e_{3}-n a$

an-da $E_{2}-a n-n a . k$ ha-ba$\mathrm{da}-\mathrm{k}\left[\mathrm{ar}-\mathrm{re}^{\text {? } \rightarrow \text { z. } 27-30.38}\right]^{39}$
„[Der Himmel] ist unser, die Erde ist unser,

aus dem Bereich des Himmels ${ }^{40}$ werde ich das E-ana auf jeden Fall rauben ${ }^{41}$ !“

an gal karede / Innana und An Z. 44

Nach vollbrachter Tat heißt es dann noch dreimal in ähnlichen Formulierungen, sie habe das E-ana geraubt ${ }^{42}$.

39 Die Verbalform ist (wie in solchen Fällen oft) defektiv geschrieben für ha-ba-da-karre<-en>.

$40 \mathrm{kar}$ wird hier im Text immer mit Komitativ konstruiert. Zu dieser Konstruktion vgl. Attinger 1993, 251 f „,enlever à qqn“ und Jagersma 2010, 452.

41 Die Aussage verwendet den in diesem Text beliebten „Affirmativ der Zukunft“. Dieser formal dem Prekativ ähnelnde Modus findet sich z. B. eindeutig in Z. 38. Auch dort steht, ebenso eindeutig wie hier, eine Form mit 1. Ps. Sg. (was kein „Prekativ“ sein kann; dafür würde Kohortativ verwendet). Die Form steht in einer Rede Utus: [eni] m nin ${ }_{9}-\hat{g}_{10}$ ma-ab-be $e_{2}$ a $\hat{g}_{26}$-e ḩa-ba-da-ab-še.g-ge - „Das Wort, das meine Schwester mir sagen wird - ich für mein Teil: ich werde es auf jeden Fall befolgen!“ Der Affirmativ der Zukunft bringt Entschlossenheit zum Ausdruck. Wer diesen Affirmativ der Zukunft verwendet, ist felsenfest überzeugt und/oder will alles daransetzen, die Aussage auch wirklich eintreten zu lassen. Ein „Affirmativ der Zukunft“ findet sich nicht in allen Darstellungen zur sumerischen Sprache, ist aber schon bei Falkenstein 1950, 121 umschrieben (,betont affirmative Bedeutung im Sinne einer bestimmt gegebenen oder eintretenden Tatsache“) und von Attinger (1993, 293 f) aufgenommen als ,affirmation catégorique“.

42 Bzw. präziser: Sie hat es herbeigeholt, vgl. Z. 153, 159 und 163, und Kapitel 5 zur TransferSprache. 


\section{Vom Himmel auf die Erde: Die Transfer-Sprache}

\subsection{Hermeneutische Differenzierung: Transfer und Transfer- Sprache}

Wenn Innana hier also „raubt, wegreißt, abreißt, wegnimmt“ (kar), dann scheint das eindeutig negativ zu sein; davon gehen die bisherigen Bearbeitungen und Studien zu diesem Epos aus. Nach van Dijk $(1998,30)$ erklärt das Epos damit, wieso die Stadt Uruk mit dem Tempel „Haus des An“ der Kultort Innanas und nicht des Himmelsgottes An war: sie habe ihn beraubt und verdrängt ${ }^{43}$. Hier muss die weitere Forschung ansetzen. Denn es bleibt unklar, wieso derselbe An Innana am Ende desselben Textes ob dieser Tat rühmt. In welcher Hinsicht dieser Raub problematisch ist, muss daher noch geklärt werden. Denn aus einer solchen negativen Formulierung zu schließen, die Tat selbst sei als negativ zu bewerten, ginge an den Eigenarten mythischer Sprache vorbei. Hier ist hermeneutische Vorsicht geboten. Drastische, negativ-schockierende Formulierungen sind typisch für die Verschriftungen mythischer Stoffe. Ihre Sprache fesselt die Aufmerksamkeit. Diese fesselnde Sprache kann in der vorliegenden Version des Mythos nicht heißen, dass Innanas Tat verurteilt würde. Denn Innana wird ja genau dieser Tat wegen am Textende höchlichst gepriesen ${ }^{44}$. Analysen von Grammatik und Lexik liefern für die Bedeutung des „Raubes“ in emischer Perspektive wichtige Indizien, von denen hier eine vorgestellt wird; weitere werden in der neuen Edition des Textes in der Reihe Mythological Studies dargestellt werden (vgl. vorläufig die Übersetzung A. Zgoll 2015).

Wichtig für die Interpretation des eigenartigen Befundes, dass Innana dem An das E-ana raubt, von demselben An aber dafür gepriesen wird, ist eine morphologische Entdeckung. Die Rede vom „Rauben“ ist in diesem Text nämlich unterschiedlich ausgestaltet. Zunächst werden die finiten Verbformen ${ }^{45}$ mit der verbalen Basis kar mit einem Separativ-Präfix $\{-\mathbf{b} \mathbf{a}-\}$ gebildet, betonen also den Aspekt, dass sich etwas wegbewegt: Das E-ana wird also weggebracht, was sich auch als „Raub“ wiedergeben lässt:

43 Vgl. auch van Dijk (1998, 11: „Das E’anna zu verlangen und zu besitzen, ist theologisch gesehen dasselbe wie sich mit An gleichstellen, ja sogar ihn zu ersetzen.“

44 Dieser Preis entfaltet sich dreifach: Zuerst spricht An Innana direkt an und sagt dabei u. a., dass sie ihn übertroffen habe (Z.144 f), dann folgt ein Preis in der 3. Person, schließlich ein abschließender Preis (z a m i m), der wieder als direkte Ansprache gestaltet ist.

45 Es gibt vier Zeilen mit finiten Verbformen der verbalen Basis kar; die anderen Stellen im Text sind infinit, so dass sie keine Morpheme für Separativ oder Ventiv enthalten. 


$\begin{array}{ll}{ }^{44} \mathrm{ha}-\mathbf{b} \mathbf{a}-\mathrm{da}-\mathrm{k}\left[\mathrm{ar}-\mathrm{re} \mathrm{e}^{? \rightarrow \mathrm{z} \cdot 27-30,38]}\right. & \begin{array}{l}\text { Innana: } \\ \text { „Ich werde das E-ana aus dem Bereich (des }\end{array} \\ & \text { Himmels) auf jeden Fall wegbringen (= } \\ \text { rauben)!“ } & \\ & \text { An: } \\ & \text { „Du (= Innana) hast es geschafft, es (= das E- } \\ & \text { ana) wegzunehmen!“ }\end{array}$

Gegen Ende des Textes hingegen finden sich in Textzeuge UnB nur noch Formulierungen mit Ventiv-Präfix $\{-\mathbf{m}-\}$, was eine Bewegung hin zu einem Zielpunkt bezeichnet:

153 und 154, UnB

「im-da-an ${ }^{1}-k$ ar-re-en

159 UnB i m - ${ }^{\top} \mathrm{d} \mathrm{a}^{\top}-\mathrm{a}$ - $-\mathrm{ka}$ r

${ }^{163}$ im-da-kar-re-en
An:

„Weil du (= Innana) dich daran gemacht hast, es (= E-ana) aus dessen (= des Himmels) Bereich herbeizuholen (auf die Erde)“

„Weil sie es (=E-ana) aus seinem (des Himmels) Bereich herbeigeholt hat“"

„Weil du (= Innana) dabei bist, es (=E-ana) aus seinem (des Himmels) Bereich herbeizuholen“

Diese Formulierungen mit Ventiv bringen eine ganz andere Nuance zum Ausdruck als die Formulierungen mit Separativ: Anstelle des „Weg-Nehmens“ steht hier das „Herbei-Holen“ im Vordergrund der Betrachtung. Fazit: kar bezeichnet im vorliegenden Text nicht nur das bösartig beraubende Weg-Nehmen, sondern auch ein positives Herbei-Holen.

\subsection{Verschiedene Perspektiven: An vs. Innana}

Der Befund führt zu neuen Fragen. Nun steht zu klären, wieso das Epos hier so verschieden formuliert. Die Antwort ergibt sich, wenn man entdeckt, dass hier zwei verschiedene Perspektiven vorgeführt werden: Der Anfang akzentuiert die Perspektive des Himmelsgottes, der nichts von seiner Macht abgeben will und dem das E-ana weg-genommen wird; später hingegen ist vom Herbei-Holen des E-ana in die Sphäre der Innana die Rede. Und diese Sphäre der Innana erweist 
sich als kongruent zur Erde bzw. zu Sumer, also zur menschlichen Sphäre ${ }^{46}$. In den Formulierungen mit Ventiv zeigt sich also die Perspektive der Innana und damit zugleich der Menschen, für die der Gewinn des E-ana eine Existenz ermöglichende Großtat ist. Der Mythos, der in diesem epischen Preislied verarbeitet ist, thematisiert mithin insbesondere den Gewinn des Tempels für die Erde und lässt sich daher gut unter der Überschrift INNANA BRINGT DAS HIMMELSHAUS FÜR DIE ERDE zusammenfassen.

Eine solch komplexe Sachlage mit klar unterschiedlichen Anliegen verschiedener Protagonisten ist auch in anderen Mythologien gut bekannt. Wie An das Himmelshaus nicht preisgeben will, so will auch Zeus das Feuer nicht den Menschen überlassen. Prometheus aber raubt das Feuer, um es den Menschen trotzdem zu bringen ${ }^{47}$. Prometheus und Innana nehmen Risiken und Opfer auf sich, um den Menschen zu helfen.

Ein Aufeinanderprallen der Interessen zeigt sich in verschiedenen Mythen der mesopotamischen und der griechischen Antike als Teil einer typischen Konstellation: Nicht nur hier versuchen jüngere Götter bzw. Heroen, den älteren etwas zu entreißen. Protagonisten wie Innana, Enki oder Prometheus sind es, die die Entwicklung der Welt vorantreiben und die Grundlagen für die Existenz der Menschen schaffen.

\section{Rekonstruktion des Mythos INNANA BRINGT DAS HIMMELSHAUS FÜR DIE ERDE}

In den vorangehenden Abschnitten ging es um die Annäherung an den Textbestand des episch gestalteten Preisliedes an gal karede / Innana und An. Auf dieser philologischen Grundlagenarbeit baut die Rekonstruktion des Mythos INNANA BRINGT DAS HIMMELSHAUS FÜR DIE ERDE auf. Durch Analyse der kleinsten handlungstragenden Einheiten, der Hyleme, und ihre weiteren Auswertungen im Gesamt der hylistischen Methodik lässt sich der mythische Erzählstoff, der im Text verarbeitet ist, in wesentlichen Teilen offenlegen ${ }^{48}$. Dabei ist allerdings

46 Texte aus dem antiken Mesopotamien wie an gal karede / Innana und An zeigen uns, wie fein und komplex die Autoren zwischen derart verschiedenen Perspektiven, derjenigen des Himmelsgottes An und derjenigen Innanas bzw. der Menschen, differenziert haben.

47 Zum Mythos Prometheus BRingt DEN MENSCHEN DAS Feuer vgl. den Beitrag von Alvensleben, Vom Himmel oder nicht vom Himmel? im vorliegenden Band.

$48 \mathrm{Zu}$ Hylemen und zur Methodik der Hylemanalyse vgl. den Beitrag von C. Zgoll, Grundlagen der hylistischen Mythosforschung (Kapitel 2) im vorliegenden Band. 
anzumerken, dass mehr als ein Drittel des Epos fehlt. Gerade bei einer solchen Herausforderung kann die Erzählstoffanalyse wesentlich zur Klärung beitragen. Aufgrund von Hylem-Überlappungen, v. a. zwischen der Schilderung von Planung und Ausführung der Planung, und durch begriffliche Bezüge lässt sich in gewissem Umfang ein Ablauf der Geschehnisse ausloten. Vieles davon darf als gesichert gelten, einiges muss hypothetisch bleiben und wird als solches kenntlich gemacht. Die Hylem-Analyse mitsamt den Zeilenangaben legt die Indizien für die Rekonstruktion offen. Die methodischen Grundlagen für diese Rekonstruktion (u. a. Extraktion der Hyleme auf Textbasis und in Reihenfolge des Textes, darauf aufbauend Rekonstruktion der stoffchronologischen Reihenfolge, Ergänzung impliziter, notwendiger Hyleme, Erfassung der durativen Hyleme etc.) werden in der monographischen Bearbeitung mit neuer Edition des Textes und ausführlichen mythologischen Analysen innerhalb der Reihe Mythological Studies dargelegt werden.

\subsection{Undeutliche Anfänge}

Der Anfang des Mythos ist bislang noch unbekannt. Festhalten lässt sich, dass INNANA BRINGT DAS HIMMELSHAUS FÜR DIE ERDE in der frühen Urzeit der Welt und Götter spielt. Dort, wo wir die Handlung des Mythos greifen können, gibt es schon Götter, aber Erde, Tag und Nacht ${ }^{49}$ und Menschheit existieren noch nicht. Die ersten mythischen Hyleme, die greifbar werden, zeigen jedenfalls, dass Innana mit ihrem Gemahl An eine Nacht verbringt (Z.41). Es handelt sich um die erste Nacht der beiden, also um ihre Hochzeitsnacht $t^{50}$.

\subsection{Hylem-Übersicht 1: Von der Hochzeit zwischen An und Innana zum Plan, das Himmelshaus zu rauben}

Die Hylem-Analyse zu den ersten erhaltenen Textpartien lautet folgendermaßen:

49 Das ergibt sich aus dem weiteren Verlauf des Mythos. Der Beginn des Epos, der sich in Fragment D nur bruchstückhaft erhalten hat, könnte dies explizit ausführen mit einer Aussage, dass es noch keine Nacht gab (Z. 4).

50 Die umfangreichen Forschungen zu diesem Punkt werden in der geplanten Monographie dargestellt werden; vgl. dazu auch S. 288 mit Anm. 51. 
- An schläft mit Innana (zum ersten Mal, d. h. in der Hochzeitsnacht) (Z. 41) ${ }^{51}$.

- $\quad$ JJetzt ist An Gemahl der Innana] (erschlossen aus Z. 41).

- [Jetzt] ist Innana Lieblingsgemahlin des An (Z. 149).

- Innana wünscht das E-ana von An (als Reaktion auf die gemeinsame Nacht, d.h. als Morgengabe) (Z. 42).

- An gibt Innana das E-ana nicht (Z. $42 \mathrm{f})$.

- Innana plant, das E-ana, d. h. den „Großen Himmel“ wegzunehmen (für die Erde) (Z. 27-30).

\subsection{Innana plant, das Himmelshaus aus dem Himmel wegzunehmen}

Um ihren Plan ins Werk zu setzen, bittet Innana ihren Bruder Utu um Unterstützung und fordert dafür unbedingten Gehorsam von ihm (Z. 31-33.45 f), den Utu ihr auch eidlich zusichert (Z. 34-39). Erst danach weiht Innana Utu in das Geschehene und in ihre Wünsche ein (Z. 41.44-47). - Auch wenn nun in der Textgrundlage 13 Zeilen fehlen (Z.48-56), so lassen sich doch aus dem später Berichteten weitere mythische Hyleme erkennen, die für diesen Teil zu erschließen sind: Jemand - es muss sich um Utu handeln - verspricht der Innana (Z.122, vgl. Z.46), dafür Sorge zu tragen, dass das E-ana vom Himmel durch den „schmalen Pfad“ herabfallen wird (Z. 122).

Mit einem weiteren Gott namens Adagbir (bzw. Adagkibir ${ }^{52}$ ) setzt Innana ihre Planungen zum Raub des Himmelshauses fort ${ }^{53}$, die dann im weiteren Verlauf des Textes in die Tat umgesetzt werden ${ }^{54}$.

51 Dass beide als ein verheiratetes Paar gedacht sind, ergibt sich aus Z. 149, wo Innana als Ans Lieblingsgemahlin bezeichnet wird. Dass es sich hier um die erste Nacht der beiden handelt, ist typisch für mythische Stoffe, die nichts Nebensächliches erzählen. Es ergibt sich überdies aus der Logik des Stoffes: da es sich um die Hochzeitsnacht handelt, hat Innana gute Chancen, dass ihr Wunsch nach dem E-ana erfüllt wird, wenn es sich nämlich um eine Morgengabe handelt; daher wird beides, die Hochzeitsnacht und der Wunsch nach dem E-ana in direkter Verbindung zueinander angeführt und ist als kausaler Zusammenhang zu verstehen (vgl. Z. 42).

52 So in Textzeuge NiC.

53 Ein erstes Gespräch mit Adagbir ist ebenfalls in der genannten Lücke Z. $48-56$ anzusetzen, dann beginnt ein zweites Gespräch (Z. 57); in dessen Kontext macht Innana etwas an der Brust des Adagbir (Z. 64).

54 Sowohl Innana wie Adagbir schildern einen Teil des Planes. - Die erhaltenen Teile des preisenden Epos zeigen, dass hier, wie in sumerischen Epen üblich, Planung und Ausführung übereinstimmen. Man schätzte es ganz offensichtlich, Passagen gleich oder ähnlich zu wieder- 


\subsection{Innana lässt das Himmelshaus nicht im Himmelsozean versinken}

Aus dem späteren Teil des Textes lässt sich erkennen, dass Innana das E-ana nicht hat sinken lassen und dass sie es vom Himmel auf die Erde geholt hat (Z. 153 f). Es handelt sich hierbei um zwei mythische Hyleme, die größere Passagen des Stoffes zusammenfassen: um Hyperhyleme ${ }^{55}$. Aus emischer Perspektive sind diese beiden Handlungen als die zentralen Eckpunkte und eigentlichen Großtaten anzusehen. Denn zuerst ist das E-ana bedroht durch böse Stürme, die es im Himmelsozean versenken. Am Ende dann gelingt es, dass das E-ana in den Bereich der Erde gelangt, obwohl mächtige Barrieren dem entgegenstehen, nämlich der kosmische Strom(gott), der die Obere von der Unteren Welt trennt, und der grauenvolle Skorpionwächter, der die Grenzen zwischen den Welten bewacht.

Im Einzelnen vollzieht sich die Handlungsabfolge folgendermaßen: Das Himmelshaus fährt über den Himmelsozean ${ }^{56}$. Der eigentliche Raub des Himmelshauses beginnt damit, dass Adagbir auf einem Magur-Schiff fährt und zwar, wie es heißt, „für das E-ana“, d.h. um es wegzubringen (Z. 66.112). An entfesselt daraufhin die bösen Winde gegen Adagbir (Z. 47.113 f), sicherlich um

holen. Gerade bei mündlichem Vortrag geht damit eine Intensivierung des Erlebens einher, was beim Publikum (auch heute noch) beliebt ist. Die Hyleme über den Raub des Himmelshauses gebe ich daher auch im Kontext der Ausführung wieder.

55 Vgl. den einführenden Beitrag von C. Zgoll, Grundlagen der hylistischen Mythosforschung, Abschnitt 2.7 im vorliegenden Band. Ausführlicher C. Zgoll 2019, 185-204, Kapitel 9.8 „Berücksichtigung stoffzusammenfassender und stoffrepräsentierender Hyleme (Hyperhylem-Funktion)“.

56 Das Himmelshaus kann selbst die Eigenschaften eines Schiffes haben, vgl. Lang 2010, 144-147. Interessant in diesem Kontext ist auch die Studie von Glassner 2002 zu den Entsprechungen der Maße zwischen dem Rettungsboot des Sintfluthelden im Gilgameš-Epos und dem Etemenanki, der Zikkurat des Marduktempels in Babylon. Alternativ kann man sich vorgestellt haben, dass das Himmelshaus auf einem Schiff fährt, z. B. einem Magur-Lastschiff. Vgl. dazu auch Abb. 2 im vorliegenden Beitrag und Wiggermann 2013, 121 mit Abb. 8b: Der Sonnengott fährt auf einem numinosen Schiff (dieses könnte für das Himmelshaus stehen); man sieht auch den Skorpion-Wächter mit erhobenen Armen. Wiggermann (2013, 127) schreibt dazu, dass „aus späteren Texten nichts von einer nächtlichen Reise der Sonne bekannt“ sei. Die im Preislied an gal karede / Innana und An vorliegende Version des Mythos INNANA BRINGT DAS HIMMELSHAUS FÜR DIE ERDE lässt den größeren Kontext erkennen, in den dieses Bild passt. Falls gemeint ist, dass der Sonnengott den Skorpionwächter schon passiert hat, ist die Fahrt unter dem Horizont, d. h. zur Nacht zu verstehen, ansonsten während des Tages. Denn erst, wenn der Sonnnengott die himmlischen Bereiche verlässt und, vorbei am Skorpionwächter, ins k ur hinabsteigt, wird es Nacht, vgl. dazu 6.8 und 6.10 . 
ihn am Raub des E-ana zu hindern. Die bösen Stürme versenken das Himmelshaus im Schilfrohr-Dickicht. Das E-ana fällt also in diesem „Schilfrohr-Dschungel“ des Himmelsozeans in die Tiefe $(Z .124 .127)^{57}$, wo es dann auf dem Himmelsfundament (Z. 130) verborgen liegt, überflutet von Wassern (Z. 118).

\subsection{Hylem-Übersicht 2: Von Innanas Plan, das Himmelshaus zu rauben, bis zum Versinken des Himmelshauses im Himmelsozean}

- Innana bittet ihren Bruder Utu um Unterstützung beim Raub des Himmelshauses (Z. 31-33).

- Innana fordert dafür unbedingten Gehorsam von Utu (Z. $45 \mathrm{f})$.

- Utu leistet einen Eidschwur, dass er Innana helfen wird (Z. 34-39).

- Innana erklärt Utu, dass An ihr das Himmelshaus nicht gegeben hat (Z. 41-43).

- Innana erklärt Utu, dass sie das Himmelshaus rauben will (Z. 41.44-47).

[13 Zeilen fehlen, Z. 48-56, doch zwei Hyleme sind erschließbar:]

- [Utu verspricht Innana, dass das E-ana vom Himmel durch den „schmalen Pfad“ herabfallen wird (Z. 122, vgl. Z. 46).

- Innana plant nun mit Adag(ki)bir den Raub des Himmelshauses ${ }^{58}$ :

$-\quad[\ldots]$

Die Einzelheiten dieser Planung werden hier im Kontext der anschließenden Ausführung des Plans dargestellt:

- [Das Himmelshaus fährt über den Himmelsozean] (implizit aus Z. 116 f).

- Adagbir fährt auf einem Magur-Lastschiff, um das E-ana wegzubringen (Z. 66.112).

- $\quad$ [An argwöhnt, dass Adagbir das E-ana rauben will.]

- [An lässt] die bösen Winde wüten gegen Adagbir. (Z. 113 f.67.47), um ihn am Raub des E-ana zu hindern.

57 Dass das Haus inmitten des Schilfrohr-Dickichts hinabfällt (und versinkt), scheint ein ganz entscheidender Punkt des Planes zu sein, denn er wird am Ende von Innanas Rede genannt und dann gleich am Anfang von Adagbirs Antwort wiederholt.

58 Ein erstes Gespräch mit Adagbir ist ebenfalls in der genannten Lücke Z. $48-56$ anzusetzen, dann beginnt ein zweites Gespräch (Z. 57); in dessen Kontext macht Innana etwas an der Brust des Adagbir (Z. 64). 
- $\quad$ Alle Schiffe (die auf dem Himmelsozean sind) sinken (Z. 115).

- Die bösen Stürme versenken das Himmelshaus im Schilfrohr-Dickicht, im Grenzbereich des Himmels, d. h. es „fällt“ hinab bis auf das Himmelsfundament (Z. 115.124.127.130).

- Dort auf dem Himmelsfundament bleibt das E-ana verborgen, überflutet von Wassern (Z. 118.130).

\subsection{Das Himmelshaus kommt aus dem Himmel(sozean) hervor, Innana bestaunt es}

Adagbir schlägt nun mit seiner Axt ins Schilfrohr (Z. 70), um das E-ana zu finden und zu retten, was ihm auch gelingt (Z. 120.125). Mit seinem großen Fangnetz rettet er im Auftrag der Innana das E-ana aus den Fluten (Z. 72.116 f). So kommt das E-ana aus den Fluten des Himmelsozeans heraus und damit „aus dem Himmel“ hervor (Z. 117, vgl. Z. 120.124.128.130). Der Hirtengott des Himmels fixiert es mit einem Seil (Z. 77.129) wie eine Kuh oder ein anderes Tier, das man an einer Leine führt, so dass es ihm nicht entrissen werden kann. Infolgedessen können die Wasser das E-ana nicht mehr überfluten (Z. 73.118), die bösen Winde können ihm nicht mehr schaden $(\mathrm{Z} .78 \mathrm{f})^{59}$. Nun umgeben udu g-Schutzgeister das E-ana (Z.130) und der Himmelsgott, weit entfernt (Z. 80), kann das E-ana nicht mehr erreichen (Z. 130). Innana aber bestaunt das E-ana (Z. 121.128).

\subsection{Hylem-Übersicht 3: Vom Auffischen des Himmelshauses bis zu dessen Entfernung aus dem Bereich des Himmelsgottes}

Auf Basis der Schilderung von Planung und Ausführung lassen sich die folgenden Hyleme des Mythos erkennen ${ }^{60}$ :

- Adagbir [schlägt] mit seiner Axt ins Schilfrohr (um an das E-ana zu gelangen) (Z. 70).

- $\quad$ Adagbir findet das E-ana (Z. 120.125).

59 Eine weitere Gottheit, deren Namen mit den Keilschriftzeichen ${ }^{d}$ HI.EN.HI.SA 6 geschrieben wird, scheint zum Gelingen beizutragen (Z. 81); sie gehört auch zu den sogenannten „Fischergöttern“, vgl. van Dijk 1998, 25 und Krebernik 2011-2013.

60 Des fragmentarischen Erhaltungszustandes wegen lassen sich nicht alle Hyleme wiedergewinnen, die in diese textliche Konkretion des Mythos eingegangen waren. 
- Adagbir rettet das E-ana mit seinem großen Fangnetz aus den Fluten (im Auftrag der Innana) (Z. 72.116 f).

- Die Fischergottheit HI.EN.Hु.ŠA macht etwas (Z. 81.118).

- Das E-ana kommt aus dem Himmel bzw. dem Himmelsfundament hervor (Z. 128.130), d. h. es kommt von da hervor, wo es unter dem Schilfrohrdikkicht versunken war (Z. 124).

- Der Hirtengott Sulazida befestigt (das E-ana) mit einem Seil (Z.129 mit Z. 45.77).

- Innana bestaunt das E-ana (Z. 121.128).

- [Sulazida übergibt das E-ana dem Utu] ${ }^{61}$.

Aus all dem ergeben sich folgende Konsequenzen:

- Das Wasser überflutet das E-ana nicht mehr (Z. 118).

- Die bösen Winde [können E-ana nicht mehr erreichen] (Z. 78 f).

- Schutzgötter umgeben das E-ana (Z.130).

- $\quad$ AAn], weit entfernt (Z. 80), kann das E-ana nicht erreichen (Z. 130).

\subsection{Innana lässt das Himmelshaus unter den Horizont und auf die Erde gelangen}

Nun ist das E-ana aus den Tiefen des Himmelsozeans aufgetaucht, innerhalb eines Schilfrohrdickichts, also in den Grenzbereichen zwischen dem Himmel und den anderen kosmischen Räumen; hier gibt es einen Übergang, einen „schmalen Pfad“ (Z.122), der aus dem Himmel herausführt ${ }^{62}$. Gegen dessen Wächter kämpft Innana (Z. 133).

Doch zuvor trinkt Innana aus dem Fluss Ulaja, einem Grenzfluss zwischen Himmel und Erde bzw. Unterwelt (Z. 132), der als numinoses Wesen vorgestellt wird und in der Verschriftung der Mythosversion im epischen Preislied an gal karede / Innana und An mit Gottesdeterminativ geschrieben ist ${ }^{63}$. Die Aussage, dass Innana aus diesem numinosen Wesen, der Grenzflussgottheit Ulaja, Was-

61 Dies lässt sich logisch erschließen: Adagbir rettet das E-ana aus dem Schilfrohrsumpf, Sulazida befestigt es mit dem Seil; später lässt Utu das E-ana durch den schmalen Pfad fallen: Dazwischen muss eine Übergabe stattgefunden haben.

62 Er erinnert an den Pfad des Sonnengottes (harrān d Šamaš) in Enūma elî̌s 5:21 (freundlicher Hinweis von B. Kärger).

63 Vgl. Frame 2014, $302 \mathrm{f}$ zum gleichnamigen Fluss in Elam, dort insbesondere die Textstellen auf S. 303; Krebernik 2014, 303 zum später bezeugten, gleichnamigen, „im neuass. Staatskult verehrten Gott“. 
ser trinkt, bleibt aufgrund ihrer Kürze kryptisch. Klar wird aus dem weiteren Verlauf, dass dieser Fluss überquert werden muss. Man bräuchte also eigentlich einen Fährmann. Auf Basis von Mythenvergleichen wird sich zeigen lassen ${ }^{64}$, dass Innana hier eine eigentlich unvorstellbare Tat vollbringt: sie trinkt nämlich das Wasser des Grenzflusses zur Unterwelt aus. Daher braucht Innana keinen Fährmann über den kosmischen Grenzstrom, weil sie ihn durch das Austrinken seiner Wasser begehbar macht. Dadurch öffnet sich für kurze Zeit ${ }^{65}$ ein Durchgang durch die Todeswasser, so dass Utu das Himmelshaus über diese gefährliche Grenze transportieren kann ${ }^{66}$.

Der Schmale Pfad, den das Himmelshaus als nächstes passieren muss, wird von dem riesigen Skorpion, einem schrecklichen numinosen Wesen, bewacht ${ }^{67}$. Es ist gewiss kein Zufall, dass der jährliche Durchgang der Sonne durch das Sternbild Skorpion (antik inkl. von Teilen des heutigen Sternbilds Waage) während der Jahrhunderte vor der uns erhaltenen Verschriftung des Textes in die Zeit des Herbstäquinoktiums fiel (ab der zweiten Hälfte des 5. Jahrtausends v. Chr., Hinweis Martin Ganter und Marco Stockhusen). D. h. um diese Zeit bezeichnet der Skorpion im Herbst die Grenzregion zwischen Himmel und Erde und wurde als Wächter der Unterwelt am Sternhimmel rekonstruiert.

Nach dem Queren des Grenzstroms zwischen Oberwelt und Unterwelt gelangt Innana zu diesem Skorpion. Sie attackiert den Skorpion und hackt ihm den Schwanz ab (Z. 133).

Der jugendliche Utu (Z. 85) hört das Signal der Innana (Z.136) und befolgt die Anweisungen Innanas, wie er es durch große Eidschwüre versprochen hatte (Z. 45 f, vgl. Z. 122). Zusammenfassend (im Format eines Hyperhylems) heißt es, dass Utu das Haus am bzw. im Horizont (ki-ša ${ }_{2}$-ra) entfernt; er bringt es also unter den Horizont (Z. 131) ${ }^{68}$; da keine weiteren Angaben gemacht werden, ist

64 Dies wird in der neuen Edition von an gal karede / Innana und An und der ausführlicheren Mythenanalyse von INNANA BRINGT DAS HIMMELSHAUS FÜR DIE ERDE ausgeführt werden.

65 Genau wie beim Skorpion, der nach dem schmerzhaften Verlust seines Schwanzes auch für kurze Zeit außer Gefecht gesetzt ist - eine Zeit, die ausreicht, um das Himmelshaus aus dem Himmel zu entführen.

$66 \mathrm{Zu}$ Utu als kosmischem Transporteur vgl. A. Zgoll 2014.

67 Dass ĝiri $i_{2}$ hier den Skorpion bezeichnet, hat van Dijk 1998, 27 ad Z. 133 erkannt. Zum Skorpion vgl. Zernecke 2008 und Pientka-Hinz 2009-2011, 577, der zufolge die Schreibung g ir i ${ }_{2}$ (so gelesen mit Attinger 2019b, 89; Pientka-Hinz liest ĝ i r $_{2}$ ) ab der altbabylonischen Zeit durch ĝ iri - $_{2}$ ab abgelöst wird. Zu Skorpionen in Wächterfunktion vgl. z. B. Gilgameš-Epos 9:38-135, Pientka-Hinz 2009-2011, 578, Wiggermann 2013, $126 \mathrm{f}$.

68 Vgl. Woods 2009, 185: "Indeed, no region of the cosmos plays upon the imagination like the horizon; seemingly approachable, but ever distant, it is the great divide between day and 
davon auszugehen, dass Utu den Transport des Himmelshauses aus dem Himmel auf die Weise durchführt, die den Rezipienten des Mythos geläufig ist. Wenn Utu etwas am Horizont entfernt, dann hat man sich vorzustellen, dass Utu am westlichen Horizont untergeht ${ }^{69}$, und dass er dabei das Himmelshaus mit sich nimmt. Utu bringt das E-ana weg, indem er es durch den schmalen Pfad am Horizont fallen lässt ${ }^{70}$ (Z. 122 mit Z. 131.135), d. h. (Utu) bringt das E-ana unter den Horizont (Z.131), also in den Bereich der Unteren Erde, der Unterwelt. Dann macht jemand, vermutlich Innana, das Himmelshaus fest (Z.135 vgl. Z. 159). Schließlich legt jemand sich oder etwas nieder (die Aussage ist nur fragmentarisch erhalten, Z. 138). Das kann bedeuten, dass Utu sich selbst in der Unterwelt niederlegt, wie es auch andernorts belegt ist ${ }^{71}$. Da es im Kontext um das E-ana geht, lässt sich dies auch so verstehen, dass Utu das E-ana dort (an einem Platz, der für es bestimmt ist ${ }^{72}$ ) niederlegt. Zugleich bedeutet dies, dass er selbst unter den Horizont gegangen ist, dass es also Nacht geworden ist.

Infolge der dramatischen Ereignisse gelingt es Innana tatsächlich, das Himmelshaus E-ana auf die Erde zu bringen (Z. 153 f.159.163). Der letzte Schritt in diesem mehrteiligen Abenteuer wird nicht mehr geschildert. Festhalten lässt sich: Wenn das Himmelshaus sich erst einmal in der Unterwelt befindet, dann kann der Himmelsgott es nicht mehr erreichen. Aus anderen Texten wissen wir, dass der Sonnengott als der Spezialist für Transporte zwischen den Welten fungiert (A. Zgoll 2014). Insofern wäre es für ihn kein Problem, das Himmelshaus Eana an den dafür bestimmten Ort auf die Erdoberfläche zu bringen, nach Sumer bzw. Uruk. Die umfassendere Auswertung von Text und mythischem Erzählstoff aber, die an anderem Ort vorgestellt wird, macht deutlich, dass gemäß der vor-

\footnotetext{
night, between what is known and what is unknown." und "As in the Egyptian conception, it is the gateway to the Netherworld" (S. 186).

69 Dass Utu untergeht, lässt sich an verschiedenen Beobachtungen ablesen: (1) Das Untergehen das Utu passt genau zu der von ihm zu erwartenden Bewegung, wenn er etwas am Horizont aus dem Himmel entfernen soll. (2) Innana hat von Utu unbedingten Gehorsam gefordert (Z. 31-33) und Utu hat den Eid geschworen, alles für Innana zu tun (erschließbar: das E-ana durch den schmalen Pfad fallen zu lassen); nach der Logik solcher Eidleistungen in Narrativen ist eine für den Eidleister nicht angenehme Konsequenz zu erwarten; diese besteht darin, dass Utu in die Unterwelt muss. (3) Damit entsteht die erste Nacht (vgl. Abschnitt 6.10).

70 „Fallen“ wird als Ausdruck des Transfers ins k u r auch anderswo verwendet, vgl. Gilgameš, Enkidu und die Unterwelt, wo die beiden hölzernen Geräte, die Gilgameš aus dem numinosen Baum hergestellt hatte, ins kur „fallen“.

71 Vgl. Lugalbanda I Z. 122 „Sobald sich unser Bruder wie (der Sonnengott) Utu, der sich schlafen gelegt hat, erheben wird“, Wilcke 2015, 233.

72 Vgl. die Zeichenspuren [... $]^{r} \mathrm{x}^{\top}-\mathrm{be}_{2} \mathrm{ba}-\mathrm{da}-\mathrm{a} \mathrm{n}-\mathrm{nu} \mathrm{u}_{2}$, was sich auch verstehen lässt als: legte er (= Utu) es (= E-ana) an dessen ... nieder.“
} 
liegenden Mythenvariante die Erde erst durch das Himmelshaus überhaupt entsteht $^{73}$. Die Geschehnisse stellte man sich folgendermaßen vor: Das Haus fällt nach unten, unter die Grenze des Himmels (den Horizont) in den Bereich der Unteren Welt (kur). Dort macht jemand es fest (Z. 135). Mit diesem fest verankerten Haus ist nun „ganz Sumer“ (Z. 156, vgl. Abschnitt 7.3) und damit auch die „Erde“ entstanden.

\subsection{Hylem-Übersicht 4: Vom Einbruch in die Untere Welt und dem Transport des Himmelshauses dorthin}

Durch die Auswertungen lässt sich die Hylem-Sequenz nun folgendermaßen festhalten:

- [Der Skorpion bewacht den Schmalen Pfad.]

- Innana trinkt das Wasser des göttlichen Grenzflusses zwischen Himmel und Unterwelt namens Ulaja aus (Z. 132).

- [Innana gelangt über den Grenzfluss Ulaja in den Bereich des kur (Gebirge und Totenreich).]

- [Jenseits des Ulaja] trifft Innana auf den [Wächter der Unterwelt (kur)], den Skorpion (Z. 133).

- Innana kämpft gegen den Skorpion (Z. 133).

- Innana schneidet dem Skorpion den Schwanz ab (Z. 133).

- [Innana macht dadurch den Schmalen Pfad frei] (erschlossen aus Z. 134+122+131).

- Innana gibt dem Utu durch Gebrüll das Signal (dass der Pfad frei ist) (Z.134).

- $\quad$ [Utu] hört das Signal der Innana (Z. 136).

- Utu gehorcht der Innana (Z.45 f):

- Utu lässt das Himmelshaus, umgeben von dessen udug-Schutzgeistern, durch den Schmalen Pfad fallen (Z. 122 mit Z. 131.135+138?). [Das bedeutet, dass Utu mit dem Himmelshaus den kurzzeitig trockenen Ulaja-Fluss quert und den Skorpion passieren kann]; d. h. [Utu] entfernt das E-ana unter den Horizont (Z. 131).

- [Utu und das Himmelshaus gelangen in den Bereich der Unterwelt (k ur).]

- [Innana?] macht das Himmelshaus fest (Z. 135) ${ }^{74}$.

- [Vermutlich ein weiteres Hylem?] (Z.137, nicht verständlich).

73 So unabhängig erwogen von A. Zgoll und G. Gabriel.

74 Parallel dazu bestimmt An in Z. 155, dass das Himmelshaus fest sein wird. 
- $\quad$ [Utu] legt sich selbst oder das Himmelshaus nieder (unter dem Horizont, im Bereich der Unterwelt (k ur)) (Z. 138).

- Das Himmelshaus erhebt sich aus der Unterwelt (kur) heraus und bildet nun Sumer und die Erde (siehe die folgenden Ausführungen).

Dass dieses Ziel erreicht wird, wird klar zum Ausdruck gebracht:

- Innana holt das Himmelshaus und damit den Großen Himmel (für die Erde) (Z. 27-30.153 f.159.163).

\subsection{Am Ende: Konsequenzen für Innana und Utu, für das Himmelshaus, für die Menschheit und die Sumerer}

Innana berichtet dem An die Neuigkeit, dass sie das E-ana auf die Erde gebracht hat (Z. 141). Als An dies vernimmt, klagt er (Z. 142 f). Seine „Klagen“ enthalten zugleich mehrere Festlegungen, dass all das für die Zukunft festgeschrieben sein soll, was Innana bewirkt hat. Damit entsprechen sie funktional Schicksalsbestimmungen.

Die Taten von Innana und die Schicksalsbestimmungen von An und Innana führen zu gewichtigen Resultaten, die für die Menschen, die Tradenten und Rezipienten des Mythos, von höchster Bedeutung sind. Formal handelt es sich um durativ-resultative Hyleme ${ }^{75}$, also um Seinsaussagen, die sich als andauernde Konsequenz aus den Geschehnissen des Mythos ergeben, die das Ziel des Mythos bezeichnen. - Als erstes benennt An die Konsequenz, die sich in seinem eigenen Verhältnis zu Innana ergeben hat:

- Innana ist durch (den Raub des) E-ana größer geworden als An (Z. 144 f).

Seine Aussage ist eine Anerkennung und Bestätigung dieser Erhöhung Innanas. In verschiedenen Varianten ziehen sich ähnliche Hyleme über die Erhöhung Innanas durch den weiteren Text, wobei sie teils aus dem Mund von An, teils als narrative Feststellungen von Erzählerseite (Z. 151 f), teils im Lobpreis auf Innana formuliert sind. Die Hyleme lauten:

- Innana hat sich selbst erhöht (Z. 151 f).

- Innana ist [daher jetzt] die große Himmelsherrin, größer als An (Z. 141+144 f).

- Innana ist größer als die Fürstengötter, die Anuna (Z. 164).

$75 \mathrm{Zu}$ durativ-resultativen Hylemen vgl. C. Zgoll, „Grundlagen der hylistischen Mythosforschung“ (Abschnitte 2.4 und 2.7) im vorliegenden Band. 
Diese Erhöhung der Innana wird schließlich als Sieg bezeichnet, den sie für die Erde errungen hat:

- Innana hat den Sieg für die Erde und damit die Macht errungen (Z. 161).

- Innana ist die bekannte Herrin (Z.161).

Dann geht es um die Entstehung der Zeit in Form von Tag und Nacht. Der Himmelsgott An bestätigt die Tat Innanas:

- An sagt:

- Innana hat Tag und Nacht mit je drei Wachen erschaffen.

- Parallel (Markierung: //) zu dieser Aussage ergeben sich weitere Konsequenzen: // Das bedeutet zugleich, sie hat das Licht, die Periode der Helligkeit, verkürzt (Z.146 f). // [Utu geht ab jetzt nachts unter.] // [Ab jetzt existieren Nacht und Tag.]

Und so geschieht $\mathrm{es}^{76}$. Die folgenden Festsprechungen des An betreffen das Himmelshaus:

- An legt fest:

- Das Himmelshaus ist fest wie der Himmel (Z. 155, vgl. Z. 159).

Dieses Festsein manifestiert sich laut Textzeuge UnB darin:

- NN zerstört das Himmelshaus nicht (Z. 155 UnB).

- Das Himmelshaus hat keinen Rivalen (Z.156 UnB) ${ }^{77}$.

Schließlich wird das Verhältnis zwischen Innana und den Menschen geregelt.

- An legt fest:

- $\quad$ Innana] wird die Menschheit erschaffen (Z. 149).

- Innana wird die Menschheit und die Sumerer zahlreich machen (Z.157 $\mathrm{UnB})^{78}$.

76 Anzumerken ist hierbei, dass Ans Spruch diese Tat der Innana zuschreibt. Textzeuge Si?A Z. 148 hingegen vermerkt, dass es so kommt, wie An es gesagt hat, schreibt die Verantwortung dafür also An zu.

77 Textzeuge Si?A überliefert eine andere Version des Mythos mit folgenden Hylemen: Das Himmelshaus entzückt NN (gemeint ist ein Gott oder Götter) immer (Z. 155 Si?A). Das Himmelshaus trägt den Namen und ist daher die „Gesamtheit der Siedlungen des Landes Sumer“ (Z. 156).

78 Textzeuge Si'A hat stattdessen die Hyleme: Innana wird [der Menschheit und den Sume-

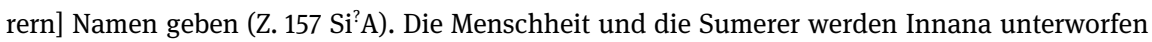
sein (Z. $157 \mathrm{Si}^{\text {? A) }}$. 
Im Vergleich mit den Festsprechungen des An sind die Festsprechungen der Innana überaus kurz, nur auf eine einzige Aussage fokussiert. Diese Aussage führt aus dem Mythos der Urzeit direkt ins Geschehen des kultischen „Heute“:

- Innana legt heute fest (Z. 160):

- Das Himmelshaus hat die Erde gut gemacht (Z. 160.163).

Der Mythos mit seiner Bewegung vom Himmel zur Erde hat hier nun sein Ziel gefunden: das Himmelshaus ist aus dem Himmel heraus- und nach unten gekommen und bildet nun eine Entität zwischen Himmel und Unterwelt, d. $h$. durch das Himmelshaus existieren nun Sumer und die Erde (vgl.6.8). Außerdem sind die Nacht und damit auch der Tag entstanden, d. h. der Sonnengott muss nun immer zwischen den Weltteilen Himmel und Unterwelt kreisen. Die noch entstehende Menschheit hat in Innana, der das Verdienst über diese Entstehungen zukommt, ihre Herrscherin und Schutzpatronin.

\section{Das Himmelshaus auf Erden: Der prototypische Charakter des E-ana}

\subsection{Implizite Identifizierung: E-ana = „Großer Himmel“}

Das mythische Epos an gal karede / Innana und An offenbart Aussagen von ontologischem Gewicht in den Details. Dort, wo der Plan Innanas von einem Raub zum ersten Mal erscheint, ist nicht vom E-ana die Rede, sondern vom „großen Himmel“. Es heißt dort, dass Innana ihren Sinn, d. h. ihre Planungskraft ${ }^{79}$ dar- $^{-}$ auf richtete, den großen Himmel wegzunehmen (Z. 27-30) ${ }^{80}$ :

${ }^{27}\left[\left(\mathrm{u}_{4} \cdot \mathrm{d}-\mathrm{bi}-\mathrm{a}\right)\left(\mathrm{di} \hat{\mathrm{g} i r^{81}}\right) \mathrm{n}\right] \mathrm{in}$ an-na- ${ }^{-} \mathrm{ke}_{4}{ }^{1}$ an gal kar-re$\mathrm{de}_{3}{ }^{\hat{\mathrm{s} e s ̌-t u}}{ }^{9} \hat{\mathrm{g} e s ̌ t u . g-g a-n i ~ b a-~}$ an-gub
27 [(Damals)] setzte [(eine Gottheit), die Herr]in des Himmels ihren Sinn darauf, den großen Himmel wegzunehmen!

79 Vgl. A. Zgoll 2020, 100-103.

80 Ein klarer intertextueller Bezug besteht hier zu an galta / Innanas Gang zur Unterwelt Z. 1-3: an gal-ta ki gal-šes ${ }^{\hat{g} e s ̌-t u}{ }^{9}$ geštu.g-ga-ni na-an-gub / diĝir an gal-ta ki

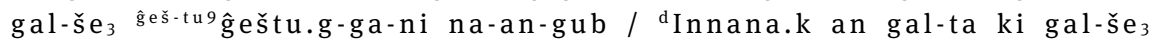
ŝeš-tug ĝeštu.g-ga-ni na-an-gub. Vor diesem Hintergrund ist auch die Ergänzung von $\mathrm{d} \mathrm{i} \hat{\mathrm{g}}$ ir in an gal karede / Innana und An Z. 27 vorgenommen.

81 Zur Ergänzung vgl. an galta / Innanas Gang zur Unterwelt Z. 2. 
${ }^{28}\left[\ldots \mathrm{ku}_{3} . \mathrm{g}\right]{ }^{\mathrm{d}}$ Innana.k-ke ${ }_{4}$ an gal kar-re-de ${ }_{3}$ seš-

${ }^{\text {tu }}{ }^{9}$ geštu.g-ga-ni ba-an-gub

${ }^{29}\left[\mathrm{an} \mathrm{su} \mathrm{u}_{3} . \hat{\mathrm{r}}-\hat{\mathrm{r}} \mathrm{a}_{2}{ }^{82} \mathrm{ša}_{3} . \mathrm{g}\right] \mathrm{an}$ -

na- ${ }^{-} a^{\top}$ an gal kar-re-de ${ }_{3}$

ĝeš-tug $\hat{g}$ eštu.g-ga-ni ba-an-

$\mathrm{gub}$

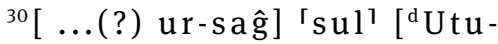

da] an gal kar-re-de 3 ĝeš-

${ }^{\text {tu }}{ }^{9}$ geštu.g-ga-ni ba-an-gub
${ }^{28}$ [... die strahlende] Innana setzte ihren Sinn darauf, den großen Himmel wegzunehmen!

29 [Vom weit entfernten Himmel, vom Inneren] des Himmels den großen Himmel wegzunehmen, darauf setzte sie ihren Sinn!

${ }^{30}$ [.... mit dem Helden], dem Jüngling [Utu] den großen Himmel wegzunehmen, darauf setzte sie ihren Sinn.

Einzig das Objekt der Handlung unterscheidet sich von den späteren Formulierungen im Text: Ist dort vom Raub des E-ana die Rede, so hier vom Raub des „großen Himmels“. Diese Variation muss eine tiefere Bedeutung haben. Impliziert wird damit etwas Erstaunliches: Das „Himmelshaus“ E-ana und der „große Himmel“ sind nicht verschiedene Entitäten, sondern zwei Seiten derselben Sache $^{83}$. Das bedeutet auch: Indem Innana das E-ana auf die Erde bringt, ereignet sich eine Tat von kosmischer Bedeutsamkeit. Der Himmel öffnet sich für die Erde, ja mehr noch: Der Himmel selbst kommt nach unten und bildet die Erde $(\text { vgl. 6.8 })^{84}$.

\subsection{Explizite Identifizierung: E-ana = „Großer Himmel“}

Dahinter verbirgt sich ein ungeheuerlicher Anspruch: Das Himmelshaus wird ja hier mit dem „großen Himmel“ identifiziert ${ }^{85}$ ! Kann der Text plausibel machen,

82 Zur Ergänzung vgl. an gal karede / Innana und An Z. 80.

83 Richter $(2003,347)$ bringt das so auf den Punkt: „Die Gleichsetzung an (- gal) = é-an-na [...] weist darauf, daß der Tempel den gleichsam manifest gewordenen ,Himmel auf Erden' wie dies auch der Name é-an-na meinen könnte - darstellt, offensichtlich in der Bedeutung eines Bindeglieds zwischen den Sphären“.

84 In der Formulierung von van Dijk (1998, 30): „Herablagerung des E’anna vom Himmel“.

85 In vielem vergleichbar und doch zugleich ganz anders ist eine thematisch ähnliche Stelle im Alten Testament gestaltet, auf die van Dijk (1998) im Motto und am Ende seines Beitrages verweist: Auf einen Traum mit der Schau von Engeln, die zwischen Erde und Himmel auf einer Treppe auf- und absteigen, reagiert Jakob so: „Und er fürchtete sich und sagte: Wie furchtbar ist diese Stätte! Dies ist nichts anderes als das Haus Gottes, und dies die Pforte des Himmels. [...] Und er gab dieser Stätte den Namen Bethel.“ (Gen 28, 17.19, Übersetzung der Elberfelder Bibel). Bet-El, was ähnlich wie E-ana „Haus des Gottes (El)“ bedeutet, ist auch hier der Zugang zum Himmel und selbst ein Stück Himmel auf Erden. Im Unterschied zur sumerischen Überlie- 
dass eine solche Transformation des Himmels gelingen kann - oder andersherum: Können Tempel auf Erden nach mesopotamischer Vorstellung tatsächlich „großer“ Himmel sein? Dem Mythos zufolge ist diese Identität nicht nur durch die Tat Innanas gesichert. Auch der Himmelsgott trägt entscheidend dazu bei, wenn er nach getaner Tat in einem performativen Sprechakt das Wesen des Tempels festsetzt ${ }^{86}$, indem er zu Innana sagt:

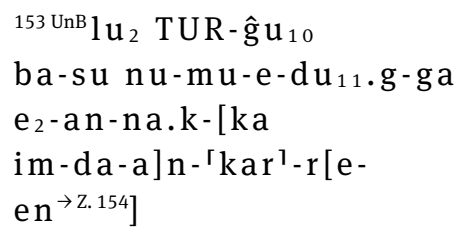

\author{
„Mein (Kleines =) Schätzchen ${ }^{87}$, \\ die du nicht zugelassen hast, dass es sinken \\ würde 88 \\ - es war (ja schließlich) das Himmelshaus ${ }^{89}$ ! \\ Weil du dich daran gemacht hast, es aus \\ dessen (= des Himmels) Bereich herbeizuho- \\ len (auf die Erde) ${ }^{90}$, \\ (o $\mathrm{du}^{91}$ ), bekannte Innana, \\ die du nicht zugelassen hast, dass es sinken \\ würde \\ - es war (ja schließlich) das Himmelshaus! \\ Weil du dich daran gemacht hast, es aus \\ dessen (= des Himmels) Bereich herbeizuho- \\ len (auf die Erde), \\ (deswegen) wird (auf jeden Fall) das Him- \\ melshaus fest sein wie der Himmel,
}

ferung von INNANA BRINGT DAS HIMMELSHAUS FÜR DIE ERDE öffnet der eine Gott JHWH hier freiwillig seine Tore und ermöglicht damit die Verbindung von Himmel und Erde.

86 Damit bestätigt er die Tat Innanas.

87 Übersetzung nach Textzeuge UnB. Text Si?A formuliert so: „[(Du), der Liebling], der nicht zugelassen hat, dass es sinken würde - es war (ja schließlich) das Himmelshaus! - du hast es geschafft, es wegzunehmen!“. Analog ist auch die folgende Zeile gestaltet.

88 Wörtlich „weil du, indem du nicht gesagt hast, Es (= das Himmelshaus) wird gesunken sein ...“..

89 In Z. $153 \mathrm{f}$ ist die Aussage „Es war das E-ana“ als Apokoinu in der Versmitte zu verstehen, d.h. auf beide Hälften des Verses zu beziehen. Dieses Stilmittel ist auch in anderen mesopotamischen Literaturwerken beliebt, vgl. bspw. Enūma elî̌s mit Gabriel 2014, 175.

90 Wörtlich „rauben“ mit Ventiv (!) zur Fokussierung auf das Ziel: „weil du dabei warst, es daraus (aus der Verfügungsgewalt des Himmels) herbei (zum Ziel) zu rauben“; zu diesen sprachlichen Feinheiten vgl. Abschnitt 5.1. Auch im Folgenden wird das Verbum ka r, wörtlich „rauben“, in der Kombination mit Ventiv „herbei“ als „herbeiholen“ übersetzt.

91 Textzeuge UnB schreibt die Verbalformen eindeutig als 2. Ps., so dass auch der Anfang der Zeile als Anrede an Innana aufzufassen ist. 
za3.g-saga ${ }_{11}$ nam-ba-an-du du $_{12}<-$ $\mathrm{du}_{12}>$ es darf auf keinen Fall eine Zerstörung geben ${ }^{92 ! “}$

an gal karede / Innana und An Z. 153-155 in der Version des Textzeugen UnB (mit Affirmativ der Zukunft, vgl. oben Anm. 41)

Mit dieser Festlegung des An sind alle Voraussetzungen erfüllt, damit aus Innanas Plan und ihrer Tat Realität wird ${ }^{93}$ : Die Identifizierung von Tempel und Himmel wird bestätigt und festgeschrieben, das E-ana wird tatsächlich zum Himmel auf Erden. Und als Himmel auf Erden wird es - so der Text - für immer Bestand haben.

\subsection{Das E-ana als Unikat und Prototyp}

Anschließend setzt An noch eine weitere wesentliche Eigenschaft des E-ana fest:

$156 \mathrm{UnB} \mathrm{gu}_{2}-\mathrm{ki} \hat{\mathrm{g}}_{2} \mathrm{kalam-ma}$ $\mathrm{mu}-\mathrm{bi} \mathrm{h} \mathrm{h}_{2}-\mathrm{Cpa}_{3} \cdot \mathrm{d}^{\mathrm{T}-\mathrm{de}} \mathrm{e}_{3}$ gaba-ri na-an-d $u_{12}-d u_{12}$
„Gesamtheit des Landes Sumer werde ich auf jeden Fall als seinen Namen (in Erscheinung bringen/ausrufen =) erschaffen! Niemals wird es dort einen Gegner haben ${ }^{94 ! “}$ an gal karede / Innana und An Z. 156

Mit dem Tempel E-ana wird hier zugleich das Land Sumer in seiner Gesamtheit, mit all seinen Siedlungen $\left(\mathrm{gu}_{2}-\mathrm{ki} \hat{\mathrm{g}}_{2}\right)$, präsent. Der Anspruch, der mit dem Eana in diesem mythischen Epos verbunden ist, ist also umfassend: Dieser Tempel ist die Keimzelle und der innerste Kern von ganz Sumer ${ }^{95}$. So würden wir

92 In Textzeuge Si’A stattdessen: „das Entzücken darüber darf dort auf keinen Fall (gewendet $=$ ) beendet werden!“

93 Es ist also nicht nur die Tat Innanas, die zum Erfolg führt, sondern auch die Bestimmung des An.

94 Wie die scheinbaren Prekative an vielen Stellen im Text als Affirmative der Zukunft aufzufassen sind (vgl. oben Anm. 41), so ist hier auch der scheinbare Prohibitiv als (negativer) Affirmativ der Zukunft zu verstehen, vgl. Attinger 1993, 290 f mit „négation catégorique“.

95 Dass Tempel und Länder zwei Seiten derselben Sache sind, zeigt sich z. B. auch in einem altbabylonischen Klagelied:

$i_{3}-d u_{3}-m_{3}$ kur in-ga-du $u_{3}-a_{3}$ 
sagen. Die originär antike Sichtweise hingegen sieht anders aus: In diesem Tempel E-ana ist ganz Sumer angelegt und insofern umgreift der Tempel ganz Sumer. Das E-ana erscheint damit als umfassende Rahmenkonstruktion einer kosmisch-globalen Weltdeutung ${ }^{96}$, die weit mehr im Blick hat, als nur einen konkreten Tempel in einer konkreten Stadt, die sich vielmehr auf das ganze Land, ja sogar auf die ganze Menschheit richtet ${ }^{97}$.

Das E-ana selbst trägt damit Unikatcharakter, insofern es auf einmalige Weise ${ }^{98}$ die Summe des Landes Sumer ist. Zugleich ist das E-ana der Himmel auf Erden und damit die prototypische Verkörperung des Präzedenzfalls ${ }^{99}$, dass Himmelshäuser auf die Erde kommen können ${ }^{100}$. Damit ist dieser Tempel tatsächlich mehr als irgendein Tempel und die Stadt Uruk ist - diesem Mythos zufolge - bedeutsamer als jede andere Stadt: Denn hier ist aus der Perspektive des Mythos InNANA BRINGT DAS HimmelShaus FÜR DIE ERDE der große Himmel herabgekommen und ist zum Anfang, Kern und Zentrum der Erde geworden.

ba-gul-gul kur ba-da-gul-gul „Als er (= Tempel) gebaut wurde, wurde auch das Land (kur) gebaut.

Er wurde vollständig zerstört - (da) wurde (auch) das Land vollständig mit ihm zerstört.“

Cohen 1981, 70, Z. 13 f, ähnlich Cohen 1988, 708 f, a+77 f; diese Textstelle verdanke ich dem Vortrag von Anne Löhnert über „Kult und Liturgie in Uruk“ beim 8. Internationalen Colloquium der Deutschen Orientgesellschaft, Berlin 26.4.2013. - In der mythischen Schöpfungserzählung CT 13, 35-38 = BM 93014 und Duplikate (vgl. Ambos 2004, 200-207) aus dem 1. Jt. v. Chr. erschafft Marduk mit den Tempeln zugleich die Städte und die Erde. Zur Austauschbarkeit von Tempel und Stadt vgl. A. Zgoll 2012a, 27-35.

96 Diese Sichtweise auf den Tempel teilt das mythische Epos auch mit der Keš-Hymne Z. 52, wo es heißt, der Tempel sei „ein Drittes neben Himmel und Erde“ (!).

97 Die letzte Festsetzung Ans bestätigt dies: Die ganze Menschheit (n a m-l $\left.u_{2}-1 u_{7}\right)$ soll Innana unterworfen sein (Z. 157).

98 Vgl. Z. 156 „Einen Rivalen wird es auf keinen Fall dort haben!“

99 In der vorliegenden Darstellung des Mythos sind zwei Prototypen zu erkennen, die absichern und begründen, wie Tempel auf die Erde kommen und die beide in Gestalt des E-ana mitgedacht werden können. Prototypisch ist erstens die Tat der Innana, die den Himmel auf die Erde holt und damit die Voraussetzung schafft, dass so etwas immer wieder gelingen kann. Prototypisch ist zweitens das E-ana selbst, insofern in ihm alle Siedlungen von Sumer mit ihren je eigenen Tempeln aufgehoben und umschlossen sind.

100 Für andere Konzepte, dass Tempel z.B. auch aus dem Süßwasserozean Abzu kommen können, vgl. Abschnitt 3.2. 


\section{Literaturverzeichnis}

Alster, B., 2004, Gudam and the Bull of Heaven, in: Dercksen, J. G. (Hg.), Assyria and Beyond, Studies Presented to Mogens Trolle Larsen, Uitgaven van het Nederlands Instituut voor het Nabije Oosten te Leiden 100, Leiden, 21-45.

Ambos, C., 2004, Mesopotamische Baurituale aus dem 1. Jt. v. Chr, Dresden.

Annus, A., 2016, The Overturned Boat: Intertextuality of the Adapa Myth and Exorcist Literature, State Archives of Assyria Studies 24, Helsinki.

Attinger, P., 1993, Eléments de linguistique sumérienne: la construction de du ul1 $_{11} / \mathrm{e} / \mathrm{di}$ „dire“, Orbis Biblicus et Orientalis Sonderband, Freiburg/Göttingen.

Attinger, P., 2006, Lesungen der Keilschriftzeichen, in: Mittermayer, C., Altbabylonische Zeichenliste der sumerisch-literarischen Texte, unter Mitarbeit von P. Attinger, Orbis Biblicus et Orientalis Sonderband, Freiburg/Göttingen.

Attinger, P., 2007, Addenda et corrigenda à Attinger dans Mittermayer 2006, in: Nouvelles Assyriologiques Brèves et Utilitaires 2007/2, 37-39.

Attinger, P., 2015, Bilgameš, Enkidu und die Unterwelt, in: Janowski, B./Schwemer, D. (Hg.), Weisheitstexte, Mythen und Epen, Texte aus der Umwelt des Alten Testaments. Neue Folge 8, Gütersloh, 24-37.

Attinger, P., 2015a, Innana und Ebih, in: Janowski, B./Schwemer, D. (Hg.), Weisheitstexte, Mythen und Epen, Texte aus der Umwelt des Alten Testaments. Neue Folge 8, Gütersloh, 37-45.

Attinger, P., 2015b, Enki und Ninchursaĝa, in: Volk, K. (Hg.), Erzählungen aus dem Land Sumer, Wiesbaden, 5-20.

Attinger, P., 2015c, Gilgameš, Enkidu und die Unterwelt, in: Volk, K. (Hg.), Erzählungen aus dem Land Sumer, Wiesbaden, 297-316.

Attinger, P., 2015d, Inanas Kampf und Sieg über Ebih, in: Volk, K. (Hg.), Erzählungen aus dem Land Sumer, Wiesbaden, 353-363.

Attinger, P., 2019, Übersetzungen [sumerischer Texte],

http://www.iaw.unibe.ch/ueber_uns/amm_amp_va_personen/prof_dr_attinger_pascal\# pane765518 (eingesehen am 2.3.2020).

Attinger, P., 2019a, Dokumente [zur Lesung der Keilschriftzeichen, zur Lexik und zur Grammatik sumerischer Texte], http://www.iaw.unibe.ch/ueber_uns/amm_amp_va_personen/prof_dr_attinger_pascal\# pane765576 (eingesehen am 2.3.2020).

Attinger, P., 2019b, Lexique sumérien-français, https://doi.org/10.5281/zenodo.2585683.

Attinger, P., 2021, Glossaire sumérien-français principalement des textes littéraires paléobabyloniens, Wiesbaden.

Attinger, P./Matuszak, J., 2015, Dumuzis Traum, in: Volk, K. (Hg.), Erzählungen aus dem Land Sumer, Wiesbaden, 399-414.

Black, J. A. u. a., 2005, 1.3.5: Inana and An, Übersetzung des Electronic Text Corpus of Sumerian Literature der Universität Oxford, http://etcsl.orinst.ox.ac.uk/cgibin/etcsl.cgi?text=t.1.3.5\# (eingesehen am 2.3.2020).

Boehmer, R. M., 2014, Ein frühnächtliches Fest zu Ehren der Stadtgöttin von Uruk, Innana, in: Zeitschrift für Orient-Archäologie 7, 127-135.

Brown, D./Zólyomi, G., 2001, "Daylight Converts to Night-Time” - An Astrological-Astronomical Reference in Sumerian Literary Context, in: Iraq 63, 149-154. 
Cancik-Kirschbaum, E., 2005, Rund-Zahlen und Ideal-Rhythmen. Beispiele aus dem alten Orient, in: Naumann, B. (Hg.), Rhythmus. Spuren eines Wechselspiels in Künsten und Wissenschaften, Würzburg, 71-91.

CDLI Literary 000342 (Inanna and An), http://cdli.ucla.edu/search/search_results.php?CompositeNumber=Q000342 (eingesehen am 2.3.2020).

Ceccarelli, M., 2012, Enkis Reise nach Nippur, in: Mittermayer, C./Ecklin, S., Altorientalische Studien zu Ehren von Pascal Attinger, OBO 256, 89-118.

Cohen, M. E., 1981, Sumerian Hymnology: The Eršemma, Hebrew Union College Annual. Supplements 2, Cincinnati.

Cohen, M. E., 1988, The Canonical Lamentations of Ancient Mesopotamia, Potomac.

Cooley, J. L., 2013, Poetic Astronomy in the Ancient Near East. The Reflexes of Celestial Science in Ancient Mesopotamia, Ugaritic, an Israelite Narrative, History, Archaeology, and Culture of the Levant 5 , Winona Lake.

Crüsemann, N./van Ess, M./Hilgert, M./Salje, B. (Hg.), 2013, Uruk. 5000 Jahre Megacity. Begleitband zur Ausstellung „Uruk. 5000 Jahre Megacity“ im Pergamonmuseum - Staatliche Museen zu Berlin, in den Reiss-Engelhorn-Museen Mannheim, Publikationen der ReissEngelhorn-Museen 58, Petersberg.

van Dijk, J. J. A., 1998, Inanna raubt den "großen Himmel”. Ein Mythos, unter Mitarbeit von A. Cavigneaux, in: Maul, S. (Hg.), Festschrift für Rykle Borger zu seinem 65. Geburtstag am 24. Mai 1994. tikip santakki mala bašmu ..., Cuneiform Monographs 10, Groningen, 9-38.

Falkenstein, A., 1950, Grammatik der Sprache Gudeas von Lagaš. Teil 2: Syntax, Analecta Orientalia 29, Rom.

Farber-Flügge, G., 1973, Der Mythos „Inanna und Enki“ unter besonderer Berücksichtigung der m e, Studia Pohl 10, Rom.

Fink, S., 2017, Inanna schreit! Kriegsgeschrei im alten Sumer, in: Gießauf, J. (Hg.): Zwischen Karawane und Orientexpress. Streifzüge durch Jahrtausende orientalischer Geschichte und Kultur. Festschrift für Hannes Galter, AOAT 434, Münster 91-98.

Frame, G., 2014, Ulai, Ulaja. A, Reallexikon der Assyriologie und Vorderasiatischen Archäologie $14,302 \mathrm{f}$.

Gabriel, G. I., 2014, Enūma eliš - Weg zu einer globalen Weltordnung. Pragmatik, Struktur und Semantik des babylonischen „Lieds auf Marduk“, Orientalische Religionen in der Antike 12, Tübingen.

George, A., 1999, The Epic of Gilgamesh. A New Translation, London/New York.

Gerstenberger, E. S., 2018: Theologie des Lobens in sumerischen Hymnen, Orientalische Religionen in der Antike 28, Tübingen.

Glassner, J.-J., 2002, L’Etemenanki, armature du cosmos, in: Nouvelles Assyriologiques Brèves et Utilitaires 2002/32, 32-34.

Haul, M., 2000, Das Etana-Epos: ein Mythos von der Himmelfahrt des Königs von Kiš, Göttinger Beiträge zum Alten Orient 4, Göttingen.

Heimpel, W., 1986, The Sun at Night and the Doors of Heaven in Babylonian Texts, in: Journal of Cuneiform Studies 38, 127-151.

Jagersma, B. H., 2010, A Descriptive Grammar of Sumerian, https://openaccess.leidenuniv.nl/handle/1887/16107.

Katz, D., 1993, Gilgamesh and Akka, Library of Oriental Texts 1, Groningen.

Kinnier Wilson, J. V., 2007, Studia Etanaica. New Texts and Discussions, Alter Orient und Altes Testament 338, Münster. 
Kirk, G. S., 1970, Myth: Its Meaning and Functions in Ancient and Other Cultures, Sather Classical Lectures 40, Cambridge/Berkeley/Los Angeles.

Krebernik, M., 2011-2013, 'ŠU.HA, Reallexikon der Assyriologie und Vorderasiatischen Archäologie 13, $258 \mathrm{f}$.

Krebernik, M., 2014, Ulai, Ulaja. B, Reallexikon der Assyriologie und Vorderasiatischen Archäologie 14, 303.

Lambert, W. G., 1980, Line 10 of the Old Babylonian Etana Legend, in: Journal of Cuneiform Studies 32, 81-85.

Lang, M., 2010, Utopie und mythische Geographie - Mesopotamisches Erbe in der syrischpatristischen Tradition am Beispiel des Paradiesberges und des Landungsplatzes der Arche, in: Voigt, R. (Hg.), Akten des 5. Symposiums zur Sprache, Geschichte, Theologie und Gegenwartslage der syrischen Kirchen, V. Deutsche Syrologentagung, Berlin 14.-15. Juli 2006, Semitica et Semitohamitica Berolinensia 9, Aachen, 137-153.

Marchesi, G., 2006, Statue regali, sovrani e templi del Protodinastico. I dati epigrafici e testuali, in: Marchetti, N. (Hg.), La statuaria regale nella Mesopotamia protodinastica. Con un'Appendice di Gianni Marchesi, Memorie dell'Accademia Nazionale dei Lincei, Serie IX, Vol. XXI, Rom, 205-271.

Matuszak, J. siehe Attinger, P.

Mittermayer, C., 2009, Enmerkara und der Herr von Arata. Ein ungleicher Wettstreit, Orbis Biblicus et Orientalis 239, Freiburg/Göttingen.

Mittermayer, C./Ecklin, S. (Hg.), 2012, Altorientalische Studien zu Ehren von Pascal Attinger.

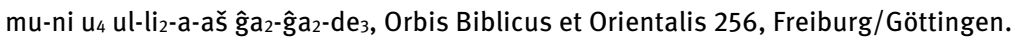

Neumann, H., 2013, Gilgamesch und Akka, in: Franke, S. (Hg.), Als die Götter Mensch waren. Eine Anthologie altorientalischer Literatur, Darmstadt u. a., 91-95.

Osten, H. H. von der, 1934, Ancient Oriental Seals in the Collection of Mr. Edward T. Newell, Oriental Institute Publications 22, Chicago.

Pettinato, G., 2001, Inanna si impadronisce del cielo, in: Pettinato, G. (Hg.), Mitologia sumerica, Turin, 252-259.

Pientka-Hinz, R., 2009-2011, Skorpion, Reallexikon der Assyriologie und Vorderasiatischen Archäologie 12, 576-580.

Richter, T., 2003, Rezension der Festschrift für Rykle Borger zu seinem 65. Geburtstag am 24. Mai 1994. tikikp santakki mala bašmu..., in: Orientalistische Literaturzeitung 98 , 345-354.

Selz, G. J., 1998, Von Treidlern, Schiff(bau)ern und Werftarbeitern, in: Archív Orientální 66, 255-264.

Waetzold, H., 2015, Gilgamesch und Akka, in: Volk, K. (Hg.), Erzählungen aus dem Land Sumer, Wiesbaden, 273-281.

Wiggermann, F. A. M., 2013, Sichtbare Mythologie. Die symbolische Landschaft Mesopotamiens, in: Zgoll, A./Kratz, R. G. (Hg.), Arbeit am Mythos. Leistung und Grenze des Mythos in Antike und Gegenwart, unter Mitarbeit von K. Maiwald, Tübingen, 109-132.

Wilcke, C., 1977, Die Anfänge der akkadischen Epen, in: Zeitschrift für Assyriologie und Vorderasiatische Archäologie 67, 153-216.

Wilcke, C., 1998, Zu "Gilgameš und Akka". Überlegungen zur Zeit von Entstehung und Niederschrift, wie auch zum Text des Epos mit einem Exkurs zur Überlieferung von "Šulgi A" und von "Lugalbanda II", in: Dietrich, M./Loretz, O. (Hg.), dubsar anta-men. Studien zur Altorientalistik. Festschrift für Willem H. Ph. Römer zur Vollendung seines 70. Lebensjahres, unter Mitarbeit von T. E. Balke, Alter Orient und Altes Testament 253, Münster, 457-487. 
Wilcke, C., 1999, Weltuntergang als Anfang. Theologische, anthropologische, politischhistorische und ästhetische Ebenen der Interpretation der Sintflutgeschichte im babylonischen Atram-hasīs-Epos, in: Jones, A. (Hg.), Weltende. Beiträge zur Kultur- und Religionswissenschaft, Wiesbaden, 63-112.

Wilcke, C., 2012, The Sumerian Poem Enmerkar und En-suhkeš-ana: Epic, Play, Or? Stage Craft at the Turn form the Third to the Second Millennium B.C. With a Score-Edition and a Translation of the Text, American Oriental Series 12, New Haven.

Woods, C., 2009, At the Edge of the World: Cosmological Conceptions of the Eastern Horizon in Mesopotamia, in: Journal of Ancient Near Eastern Religions 9, 183-239.

Zernecke, A. E., 2008, Warum sitzt der Skorpion unter dem Bett? Überlegungen zur Deutung eines altorientalischen Fruchtbarkeitssymbols, in: Zeitschrift des Deutschen PalästinaVereins 124, 107-127.

Zgoll, A., 2006, Traum und Welterleben im antiken Mesopotamien. Traumtheorie und Traumpraxis im 3. - 1. Jt. v. Chr. als Horizont einer Kulturgeschichte des Träumens, Alter Orient und Altes Testament 333, Münster.

Zgoll, A., 2010, monumentum aere perennius - Mauerring und Ringkomposition im GilgamešEpos, in: Shehata, D./Weiershäuser, F./Zand, K. V. (Hg.), Von Göttern und Menschen. Beiträge zur Literatur und Geschichte des Alten Orients. Festschrift für Brigitte Groneberg, Cuneiform Monographs 41, Leiden/Boston, 443-470.

Zgoll, A., 2012, Inanna - Stadtgöttin von Uruk, in: Crüsemann, N./van Ess, M./Hilgert, M./Salje, B. (Hg.), Uruk: 5000 Jahre Megacity; Begleitband zur Ausstellung „Uruk - 5000 Jahre Megacity” im Pergamonmuseum - Staatliche Museen zu Berlin, in den ReissEngelhorn-Museen Mannheim, Publikationen der Reiss-Engelhorn-Museen 58, Petersberg, 71-79.

Zgoll, A., 2012a, Welt, Götter und Menschen in den Schöpfungsentwürfen des antiken Mesopotamien, in: Schmid, K. (Hg.), Schöpfung, Themen der Theologie 4, Stuttgart, 17-70.

Zgoll, A., 2014, Der Sonnengott als Transporteur von Seelen (Psychopompos) und Dingen zwischen den Welten im antiken Mesopotamien. Mit einem Einblick in den konzeptuellen Hintergrund des taklimtu-Rituals, in: Koslova, N./Vizirova, E./Zólyomi, G. (Hg.) Studies in Sumerian Language and Literature. Festschrift für Joachim Krecher, Babel und Bibel 8, Orientalia et Classica 56, Winona Lake, 617-633.

Zgoll, A., 2015, Innana holt das erste Himmelshaus auf die Erde, in: Janowski, B./Schwemer, D. (Hg.), Weisheitstexte, Mythen und Epen, Texte aus der Umwelt des Alten Testaments. Neue Folge 8, Gütersloh, 45-55.

Zgoll, A., 2015a: Nin-me-šara - Mythen als argumentative Waffen in einem rituellen Lied der Hohepriesterin En-hedu-Ana, in: Janowski, B./Schwemer, D. (Hg.), Weisheitstexte, Mythen und Epen, Texte aus der Umwelt des Alten Testaments. Neue Folge 8, Gütersloh, 55-67.

Zgoll, A., 2020, Durch Tod zur Macht, selbst über den Tod. Mythische Strata von Unterweltsgang und Auferstehung der Innana/Ištar in sumerischen und akkadischen Quellen, in: Zgoll, A./Zgoll, C. (Hg.), Mythische Sphärenwechsel. Methodisch neue Zugänge zu antiken Mythen in Orient und Okzident, Mythological Studies 2, Boston/Berlin, 83-159.

Zgoll, A., 2020a, Condensation of Myths. A Hermeneutic Key to a Myth about Innana and the Instruments of Power (me), Incorporated in the Epic angalta, in: Sommerfeld, W. (Hg.), Dealing with Antiquity - Past, Present, and Future, Proceedings der 63. Rencontre Assyriologique Internationale Marburg 2017, Alter Orient und Altes Testament 460, Münster, 427-447. 
Zgoll, A., 2021, Innana Conquers Ur. A Myth for Mutual Empowerment, Created by En-hedu-ana, in: Droß-Krüpe, K./Fink, S. (Hg.), (Self-)Presentation and Perception of Powerful Women in the Ancient World. Proceedings of the 8th Melammu Workshop, Kassel 31 January - 1 February 2019, Münster, 11-53.

Zgoll, A./Zgoll, C., 2020, Innana-Ištars Durchgang durch das Totenreich in Dichtung und Kult. Durch Hylemanalysen zur Erschließung von Spuren mythischer Stoffvarianten in kultischer Praxis und epischer Verdichtung, in: Arkhipov, I./Kogan, L./Koslova, N. (Hg.), The Third Millennium: Studies in Early Mesopotamia and Syria in Honor of Manfred Krebernik and Walter Sommerfeld, Cuneiform Monographs 50, Leiden, 749-801.

Zgoll A./Zgoll C. (Hg.), 2020a, Mythische Sphärenwechsel. Methodisch neue Zugänge zu antiken Mythen in Orient und Okzident, Mythological Studies 2 (https://doi.org/10.1515/ 9783110652543), Berlin/Boston.

Zgoll, C., 2019, Tractatus mythologicus. Theorie und Methodik zur Erforschung von Mythen als Grundlegung einer allgemeinen, transmedialen und komparatistischen Stoffwissenschaft, Mythological Studies 1 (https://doi.org/10.1515/9783110541588), Berlin/Boston.

Zgoll, C., 2020, Myths as Polymorphous and Polystratric Erzählstoffe: A Theoretical and Methodological Foundation, in: Zgoll, A./Zgoll, C. (Hg.), Mythische Sphärenwechsel. Methodisch neue Zugänge zu antiken Mythen in Orient und Okzident, Mythological Studies 2, Boston/Berlin, 1-82.

Zgoll, C. siehe Zgoll, A.

Zólyomi, G., 2000, W 16743ac (= AUWE 23 101), in: Nouvelles Assyriologiques Brèves et Utilitaires $2000 / 2,41 \mathrm{f}$.

Zólyomi, G. siehe Brown, D. 
